\title{
DIE \\ ETRUSKISCHE BRONZELEBER VON PIACENZA
}

IN IHRER SYMBOLISCHEN BEDEUTUNG

\author{
EIN VERSUCH
}

VON

W. von BARTELS

MIT ZWEI ABBILDUNGEN

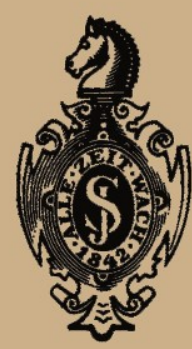

BERLIN

VERLAG VON JULIUS SPRINGER 1910 


\section{DIE}

\section{ETRUSKISCHE BRONZELEBER VON PIACENZA}

IN IHRER SYMBOLISCHEN BEDEUTUNG

EIN VERSUCH

VON

W. von BARTELS

MIT ZWEI ABBILDUNGEN

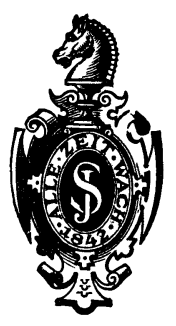

BERLIN

VERLAG VON JULIUS SPRINGER 1910 
ISBN 978-3-642-51278-0

ISBN 978-3-642-51397-8 (eBook)

DOI 10.1007/978-3-642-51397-8

Alle Rechte, insbesondere das der

Ubersetzung in fremde Sprachen, vorbehalten. 


\section{Einleitung.}

Gern komme ich der Aufforderung der geehrten Verfasserin entgegen, ihrer interessanten und anregenden Abhandlung einige Geleitworte mitzugeben, um so mehr, da ich zuerst der Untersuchung skeptisch gegenüberstand und erst durch tieferes Eindringen mich von der Richtigkeit der Hauptthese der Arbeit überzeugte, daß nämlich in der etruskischen Bronzeleber eine verwickelte Symbolik steckt.

Ich bin zwar nicht Etruskologe und darf mir daher über das, was spezifisch ins Etruskische hinübergreift, kein Urteil erlauben. Die Arbeit geht aber weit über den Rahmen des Etruskischen hinaus und berührt meine eigenen Forschungen über die Leberschau in der antiken Welt so nahe, daß ich es wagen darf, auf Grund meiner eingehenden Beschäftigung in den letzten drei Jahren mit diesem Thema, an die Fachgenossen zu appellieren, die Arbeit der geehrten Verfasserin einer wohlwollenden Prüfung $\mathrm{zu}$ unterziehen und zwar gerade, weil sie neue Gesichtspunkte eröffnet. Die Verfasserin geht ihre eigenen Wege und befolgt eine Methode, die wohl zuerst befremden mag, aber wenn man sich die Mühe gibt näher zuzusehen, so wird man sich davon überzeugen, daß sie an bereits gewonnene Resultate anknüpft. So vor allem an eine Verbindung zwischen der Leberschau und der antiken Himmelsschau und Himmelskunde, die ja durch die Einteilung des Randes auf der etruskischen Bronzeleber in 16 Regionen - entsprechend den 16 Regionen des Himmels bereits äußerlich kenntlich gemacht wird und durch die von mir nachgewiesene Verknüpfung zwischen der Himmelsschau und Leberschau bei den Babyloniern weiter beleuchtet wird ${ }^{\mathbf{1}}$ ).

Geht man von dieser Verbindung aus, so ist man gewiß zu der Annahme berechtigt, daß man sowohl in Etrurien wie in Babylonien sich auf dem besten Wege befand, zur weiteren Spekulation über das Wesen der beiden Divinationslehren fortzuschreiten. Erscheint

1) Siehe des Verfassers zwei Abhandlungen: „The Sign and Name for Planet in Babylonian" und "Hepatoscopy and Astrology in Babylonia and Assyria" in den Proceedings of the American Philosophical Society. Bd. 47. S. $105-129$ und S. $646-676$. 
die Leber nach antiker Auffassung als Sitz des Lebens, als ein „Abbild des Weltganzen im Kleinen“, wie $\mathrm{K}$ örte ${ }^{1}$ ) sich treffend ausdrückt, so wird man auch den Versuch gemacht haben, die Bestandteile der Leber als Wiederspiegelung der Himmelskörper auszulegen; und in der weiteren Ausführung dieser Idee gelangt man durch die natürliche Herbeiziehung der herrschenden Kosmologie in das Gebiet der mystischen Symbolik, oder in anderen Worten, zu einer Art Kabbala, genau wie sich in Ägypten, im Judentum und Christentum und im Islam eine mystische Lehre auf dem Boden der religiösen Doktrin entfaltete.

In meinen Studien über die babylonische Divinationslehre - und besonders in der Astrologie - glaube ich Spuren einer mystischen Auslegung der Himmelserscheinungen in Verbindung mit der herrschenden Weltanschauung gefunden zu haben, über die ich, sobald es meine Zeit erlaubt, an anderer Stelle handeln werde. Selbst wenn man nicht geneigt ist, so weit zu gehen, wie Winkler und J eremias, so muß man doch anerkennen, daß die Ausführungen dieser Gelehrten über das, was sie die "Altorientalische Weltanschauung" bezeichnen, den Beweis für eine mystische Lehre, die sich auf dem Boden der Babylonisch-Assyrischen Religion entfaltet hat, geliefert haben, und selbst Zimmern ${ }^{2}$, obwohl er sich sehr vorsichtig ausdrückt, ist dieser Auffassung nicht abgeneigt.

Ja man darf es als allgemein geltenden Grundsatz aufstellen, daß ein hoher Grad von Kultur stets von einer Neigung zu einer mystischen Weltauffassung und zu einer mystischen Symbolik im Kult begleitet wird. Es ist daher von vornherein anzunehmen, daß ein gebildetes Volk, wie es die alten Etrusker sicher waren, denselben Weg betreten.

Selbst wenn manches oder sogar vieles, das die geehrte Verfasserin in ihrem Versuch, die Symbolik der Bronzeleber zu deuten, aufstellt, sich als irrig herausstellen sollte, so bleibt doch ihre Hauptthese, daß in diesem merkwürdigen Objekt eine Symbolik steckt, und zwar eine Wiederspiegelung der herrschenden Weltanschauung, bestehen.

Jedenfalls verdienen die Untersuchungen, die in der vorliegenden Abhandlung niedergelegt sind, gehört und geprüft $\mathrm{zu}$ werden.

Was das Etruskische betrifft, so sollten gerade die Etruskologen, die ja selbst anerkennen, daß man bis jetzt nur geringe Fortschritte in der Lösung des etruskischen Problems gemacht hat ${ }^{3}$ ),

1) "Die Bronzeleber von Piacenza“ in dẻn Mitteilungen des Kaiserl. Arch. Instituts, Römische Abteilung. Bd. XX. S. 36. Man lese die ganze Stelle nach, die als Grundlage für eine symbolische Deutung gelten kann.

״) „Keilinschriften und das Alte Testament", Abschnitt „Marduk“ (S. 370 bis 396). Siehe besonders die Anmerkung auf S. 377.

3) Herbig, Zum heutigen Stand der etruskischen Frage (München 1907). Separatabdruck aus der Beilage zur Münchener Allgemeinen Zeitung; sowie desselben Verfassers „Indogermanische Sprachwissenschaft und Etruskologie“ (Indogermanische Forschungen XXV. S. 360 ff.) 
bereit sein, ohne Vorurteil an die Arbeit der Frau von Bartels heranzutreten. Es ist gewiß ein Wagnis - und die Verfasserin ist sich dessen wohl bewußt - an Stelle der von den Etruskologen angenommenen Identifizierungen der Wörter der Inschriften auf der Bronzeleber mit etruskischen Göttern, auf die Begriffe zurückzugehen, woraus die Bezeichnungen der Götter sich entwickelten; aber von vornherein abzuweisen ist diese These nicht.

Gelingt es mit Hilfe dieser Hypothese die Symbolik der Bronzeleber zu erschließen, so ergibt sich die Möglichkeit, daß man mit den Götternamen mystische Spielereien trieb, wofür es ja auch bei anderen Völkern Beispiele gibt.

Man prüfe also ohne Vorurteil! Es wäre ja nicht das erste Mal in der Geschichte der Wissenschaft, daß ein Forschungsgebiet von einem außerhalb des engeren Fachkreises Stehenden einen wertvollen Anstoß erhalten hat.

Philadelphia, im Januar 1910. 
Einerseits ist es ein Wagnis, den Arbeiten über die Bronzeleber von Piacenza eine neue hinzuzufügen; andererseits ist es natürlich, daß diese Versuche sich folgen müssen, bis über das Resultat kein Zweifel mehr bestehen wird.

Seit der Entdeckung der, ,jetzt berühmten Bronzeleber von Piacenza“, wie Jas trow in seinem Werk: „Die Religion Babyloniens und Assyriens" (II. S. 219) sie nennt, welche im Jahre 1877 stattfand, hat sich eine ganze Literatur um dieses merkwürdige Altertumsstück gesammelt; und zwar befassen sich die vielen Arbeiten, die von Gelehrten verschiedener Länder der Erforschung der Bronzeleber gewidmet sind, einerseits mit der Feststellung des eigentlichen Charakters des Objektes, andererseits mit der Erklärung der Inschriften.

Auf eine Geschichte dieser Untersuchungen einzugehen, würde zu weit führen, und ist auch für den Zweck, den wir im Auge haben, nicht nötig. Es genügt, darauf aufmerksam zu machen, daßs man glaubte - so De ecke —, daß man die Darstellung eines Templums vor sich hatte; aber man erkannte bald, daß das irrig war und daß das Stück als eine Leber - wohl eine Schafsleber - so Deecke (Etrusk. Forschungen II) und Körte - zu betrachten sei. Aber nicht $\mathrm{n}$ ur eine Leber, sondern wie Ja strow sagt: (s. Sign and Name for Planet in Babylonian S. 147) ", m microcosm reflecting the macrocosm".

Auffallend ist bei der Leber der Umstand, daß sich an deren, in 16 Teile eingeteiltem Rande, sowie im inneren Raume - ebenfalls offenbar nach einem Schema eingeteilt - etruskische Worte befinden, die gewiß zum großen Teil Götternamen oder Götterattribute darstellen. Mit diesen Namen und Attributen befaßs sich Thulin in einer vor wenigen Jahren erschienenen Schrift ${ }^{1}$ ), in der er einen $\mathrm{Zu}$ sammenhang zwischen den 16 Teilen des Leberrandes und den 16 Götterregionen, die wir durch Martianus Capella kennen gelernt haben, nachzuweisen sucht.

Ohne hier auf die Frage einzugehen, ob sich Thulins These behaupten läßt, so muß doch von allen Seiten zugegeben werden, daß die Leber eine Verbindung von der Leberschau mit kosmischen

1) Die Götter des Martianus Capella und der Bronzeleber zu Piacenza (Göteborg 1906). 
Vorstellungen ist. Ist dem so, so dürfen wir einen Schritt weiter gehen und annehmen, daß die Leber als ein Symbol aufzufassen sei. In anderen Worten: Während die Teile der Leber wirklich dargestellt sind, wenn auch in sehr schematischer Form, so müssen diese Teile zugleich in Verbindung gebracht werden mit etruskischer Götterlehre, so daß ein Zusammenhang zwischen dem Namen oder Attribute eines Gottes und dem Teile der Leber, auf dem sich die betreffende Schreibung findet, gewiß nicht ausgeschlossen ist. Handelt es sich aber um eine Symbolik, so ist die Frage nur, wie einfach oder wie kompliziert eine solche Symbolik ist.

Ich bin nach reiflichem Studium zu der Überzeugung gelangt, daß man mit der Annahme einer einfachen Symbolik nicht zu einer befriedigenden Erklärung der Leber durchdringen kann. Stellt die Bronzeleber eine Welt im kleinen dar, so muß sie soweit als möglich ein Abbild der ganzen Weltvorstellung sein, wie sie sich unter den etruskischen Priestern im Laufe der Zeit entwickelt hatte; und deshalb glaube ich, daß der rechte Weg zur Lösung der Bedeutung der Bronzeleber nur durch die Annahme einer komplizierten Symbolik betreten werden kann.

Schon das Äußere der Leber scheint darauf hinzuweisen und zwar zuerst durch die Art der Darstellung. Es ist festgestellt, daß die Bronzeleber von Piacenza nicht sehr alt ist (s. Thulin "Götter des Mart. Cap. S. 42, "sicher nicht älter, als das 3. Jahrh. v. Chr.“); sie ist also $\mathrm{zu}$ einer Zeit entstanden, als die Handgeschicklichkeit der Bildhauer, Former und Gießer vollkommen ausgebildet war, und die naturalistische Darstellung irgend eines Gegenstandes technische Schwierigkeiten nicht $\mathrm{zu}$ fürchten brauchte. Wenn trotzdem die Abteilungen und Erhöhungen der wirklichen Leber auf dieser Bronzeleber so dargestellt sind, daß sie einerseits die Wirklichkeit nicht ganz verleugnen, andererseits aber auf andere Dinge hinzuweisen scheinen, so muß dieses Absicht sein.

Man drückte, schon ehe Worte in die einzelnen Abteilungen hinein graviert wurden, aus, daß dieser Bronzegegenstand keine „Leber an sich" sondern eine „Leber zum Zwecke" dessen und dessen sei und änderte deshalb die Wirklichkeit zweckentsprechend ab.

Diese beabsichtigte Doppelsinnigkeit macht es einerseits verständlich, daß die Gelehrten - so De e cke - einmal ein Templum, und einmal ( $\mathrm{P}$ og gi) ein Amulet neben der Darstellung einer Leber ${ }^{\mathrm{l}}$ ) in der Bronze entdeckten; andererseits zwingt eben die beabsichtigte Doppelsinnigkeit den Beschauer dazu, in diesem schematisierten Processus caudatus etwa einen "Finger" oder "einen Berg“; in diesem schematisierten Processus papillaris etwa "eine Zunge"; in dieser schematisierten Gallenblase etwa eine "Keule“ zu sehen;

1) Deecke, Etr. Forsch.-Stud. II, 66 (1882) und Körte, Die Bronzeleber von Piacenza, Mitteil. d. Kaiserl. Deutsch. Archäol. Inst. XX. S. 348-379. 
oder in dem umrissenen Mittelpunkte der kleineren (südlichen) Leberhälfte etwa einen „Brunnen“. Einer realistisch dargestellten, also "wirklichen" Leber gegenüber würden niemandem solche Vergleiche einfallen, wohl aber dieser "unwirklichen“ gegenüber, weil sie als eine einen besonderen Sinn oder besonderen Gedanken enthaltende Leber dargestellt ist.

In welcher Richtung dieser in der symbolischen Leber ausgedrückte Sinn liegt, stellen die auf derselben verteilten Worte fest, welche den bekannten und durch die Inschriften auf den etruskischen Spiegeln unbezweifelbar festgestellten Götternamen zu ähnlich sind, um sich weit davon $\mathrm{zu}$ entfernen.

Aber auch dem unbefangensten Beschauer muß es auffallen, daß die Worte von der Leber den Götternamen zwar ähnlich, aber ảoch nicht sie selber sind. So steht zwar auf der Leber zweimal "tin“, einmal „tins" und einmal „tins 9 “, aber kein einziges Mal das unbezweifelbar für Juppiter gebrauchte tina oder tinia; so steht auf der Leber ne $\mathcal{f}$, aber nicht das unbezweifelbar für Neptun gebrauchte neףuns; so steht auf der Leber mar und mari aber nicht das für Mars gebrauchte maris; so steht auf der Leber herc, aber nicht das für Herkules gebrauchte hercle, heracle; so steht auf der Leber selva, aber nicht das für Silvanus gebrauchte selvans.

Daß dieses lauter Abkürzungen sein sollen, wie man annimmt, (so Thulin) erscheint bei einem so heiligen Gerät, wie es die Leber darzustellen scheint, nicht einleuchtend, besonders, da man ja nur die abteilenden Linien, welche einzelnen Worten übermäßig viel Raum geben, hätte zu ändern brauchen; ganz abgesehen davon, daß z. B. neben ne $\mathcal{\vartheta}$ noch leerer Raum für einen oder zwei Buchstaben, auch bei der gegenwärtigen Raumverteilung, vorhanden ist. Wenn Abkürzungen für Götternamen überhaupt zulässig gewesen wären, so dürfte man dieselben auch anderswo, z. B. auf den Spiegeln, erwarten. Das ist aber nicht der Fall.

Man könnte hier einwenden, daß z. B. bei den Römern und auch sonst abgekürzte Formen, oder monogramm- oder siegelartige Zusammenziehungen von Götternamen gebräuchlich waren, wie sie Traube zusammengestellt hat. Da es sich aber hier um ein etruskisches Dokument handelt, so ist es gewiß erlaubt, die etruskischen Spiegelinschriften dafür anzuziehen, daß sich dortselbst keine $\mathrm{Ab}$ kürzungen für Götternamen vorfinden. Wenn aber Scheu vor dem Aussprechen heiliger Namen oder andere Gründe auch bei den Etruskern Abkürzungen von Götternamen zur Gewohnheit gemacht haben würden, so wäre solche Gewohnheit mit Wahrscheinlichkeit auf den Spiegeln zum Ausdruck gelangt.

Eine natürlichere Erklärung scheint es, in tin, ne $\mathcal{Y}$, here usw. diejenigen Worte anzunehmen, aus denen sich die Namen der betreffenden Götter entwickelt haben könnten. 
Kein Philologe kann annehmen, daß tin, tins, tins 9 , tina und tinia ein und dasselbe Wort mit derselben Bedeutung sein kann. Das erscheint völlig ausgeschlossen. Dagegen ist es klar, daß tin, tins, tins $\vartheta$, tina und tinia Formen e in er Wurzel und Formen einer Bedeutung darstellen; und da die unbezweifelbar festgestellten Götternamen sich auf der Leber nicht vorfinden, mit Ausnahme von fufluns = Dionysos und usil = Sonnengott, und folglich auf der Leber nur von diesen beiden unbezweifelbar festgestellten Göttern die Rede ist, während im übrigen sich Worte vorfinden, welche den Götternamen nur ähnlich sind, so liegt die Annahme nahe, in diesen Worten diejenigen göttlichen Eigenschaften zu sehen, aus denen sich die Personifikationen der betreffenden Götter später entwickelt haben.

Der gegenwärtige Stand der etruskischen Frage ist ganz dazu angetan, neuen Erscheinungen auf diesem Gebiete einen dornenvollen Weg zu bereiten. Man ist durch die massenhaften Deutungsversuche, welche alle nach kurzer Zeit aufgegeben werden mußten, sehr skeptisch geworden; und das mit Recht. So gut aber Gifte Heilwirkungen enthalten, so gut enthalten auch Irrtümer Wahrheiten; deshalb sollte jeder Neuerscheinung, auch wenn sie Irrtümer enthält, das Recht gehört zu werden zugebilligt werden. Zeit und Erfahrung sondern Wertloses unerbittlich aus.

Es ist hier nicht der Platz, auf eine Worterklärung einzugehen, da dieser gegenwärtige Versuch nur der Erklärung der Leber in ihrer symbolischen Bedeutung gilt. Nur soviel sei hier gesagt: so lange man versucht, etruskische Worte mit denen einer ähnlich klingenden Sprache aufzulösen, wird man stets nach einer gewissen Zeit festsitzen, weil etruskisch nur etruskisch sein kann und nicht lateinisch, oder litthauisch, oder sonst eine Sprache.

Noch eine andere Bemerkung: Wir würden uns wundern, wenn jemand den Ulfilas und die Worte aus den Münchener Neuesten Nachrichten, oder den Roman de Rou und die Worte aus dem Figaro, oder Chaucer und die Worte aus der Times, weil es jedesmal die Worte einer Sprache sind, auf dieselbe Weise entziffern wollte. Warum tut man es beim etruskischen und nennt „Wechseln von Buchstaben" was „Lebensgang der Sprache ist?" Auch das Etruskische hat seine Jugend, seine Lebenshöhe und sein Alter.

Mehr darüber hoffe ich in einer anderen Arbeit zu geben.

Der augenblicklich bestehenden Skepsis allen etruskischen Erklärungsversuchen gegenüber Rechnung tragend, will ich versuchen eine symbolische Deutung der Leber von Piacenza zu geben, ohne auf eine Worterklärung einzugehen, außer wo dieses ganz unerläßlich ist. Nur soviel sei hier bemerkt: Wenn wir ne 9 uns ganz unanfechtbar als das etruskische Wort für Neptun kennen und wir dazu auf der Leber ne $\boldsymbol{\vartheta}$ finden, so ist doch die Idee nicht völlig von der Hand zu weisen, daß dieses ne $\boldsymbol{\vartheta}$ diejenige Eigenschaft ausdrücken 
könnte, welche später zu einem persönlichen Gott geworden ist; also etwa "naß", weil Neptun die Personifikation des Nassen ist. Oder wenn wir hercle (und heracle) unangreifbar als das etruskische Wort für Herakles kennen, (einmal her le s. De ecke, Etrusk. Forsch. IV. S. 74), daß das herc der Leber diejenige Eigenschaft ausdrücken könnte, als deren Personifikation Herakles zu gelten hat: als die vom Gotte erzeugte, von der Erde geborene Kraft, also etwa „erdig, irdisch" 1).

Dasselbe muß bei tin der Fall sein. Wir kennen tina und tinia ganz unbezweifelbar als das etruskische Wort für Juppiter; wir wissen aber ebenso unbezweifelbar, daß die Etrusker den "Sonnengott" auf den Spiegeln nicht tinia, sondern mit dem auf der Oberseite der Leber stehenden Worte „usil" bezeichneten; folglich kann der etruskische tinia nicht Juppiter im römischen Sinne als "Sonnengott" sein, weil dafür die andere Benennung „usil“ vorliegt; sondern tinia-Jupiter muß den Etruskern eine Form, oder Abzweigung von derjenigen Vorstellung des Sonnengottes, die sie mit „usil“ bezeichneten, gewesen sein.

Nehmen wir die griechische Vorstellung von Zeus als Sohn des Kronos, also als Sohn eines vor Zeus vorhandenen Gottes als Erklärung zu Hilfe, so kommen wir, ganz ohne Etymologisierung, darauf: daß tinia im Verhältnis zu usil, der die Sonne, das Sonnenlicht par excellence ist, etwa als dessen jüngere, im Wirkungskreis beschränktere Ausgabe, etwa als sein Sohn, oder als Verwalter oder Vermittler des Lichtes, als Verwahrer und Ausgeber desselben, d. i. wohl als "Tag“" auch im Etruskischen zu gelten habe. Da auf der Leber nicht von einem Gotte tinia die Rede ist, sondern nur tin, tins und tins $\vartheta$ vorkommen, so müssen diese Worte das Teilen, Vorführen oder Verwalten des ewigen Sonnenlichtes par excellence, dessen Inhaber unbezweifelbar usil ist, betreffen.

Diese Erklärungen haben mit der etruskischen Frage nur insofern zu tun, als die besprochenen Worte zufällig der etruskischen Sprache angehören. An sich sind die obigen Ausführungen nichts weiter, als festgestellte Tatsachen, ohne Bezug auf Wortforschung.

1) Da für den Sonnengott sowohl, als für Dionysos echt etruskische Worte ohne Anklang an griechische Überlieferung verbürgt sind, so ist es unwahrscheinlich, gerade für Herkules, der als Vater des Stammvaters der Etrusker ihnen besonders nahe steht, die einfache Übernahme des griechischen Namens anzunehmen; ich glaube, daß „hercle" (jünger „her $\chi \mathrm{le}^{\text {") }}$ als echt etruskisches Wort betrachtet werden muß, aus herc entstanden, wie ne $\vartheta$ uns aus ne $\vartheta$, tinia aus tin usw. Die Erklärungen der alten Schriftsteller (Diod. IV, $10 \delta \iota^{\prime}{ }^{\prime} H \varrho \alpha \nu$

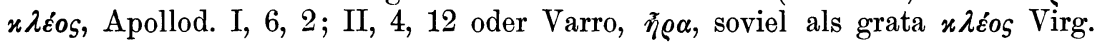
Aen. VII, 564) weisen darauf hin, daß zu ihren Zeiten schon nach Erklärungen des Wortes gesucht werden mufte. Ich glaube, wie gesagt, daf dem etrusk. herc eine Wurzel era oder ere zugrunde liegt. S. auch Mommsen, Unterital. Dial. S. 262 und Preller, Röm. Mythol. S. 647, vor allem aber Herodot II. 43,44 . 
Enthält die Leber wirklich eine Anzahl von Worten, aus denen sich die Götter in menschlicher Form erst entwickelt hätten, so drängt sich der Gedanke auf, daß der Inhalt der Leber aus einer Zeit stammt, welche weiter zurückliegt, als das Auftreten persönlich gedachter Götter, so daß also zwischen dem Alter der vorliegenden Bronze (nach Thulin oben „nicht älter als das dritte Jahrh. v. Chr.") und ihrem Inhalt eine große Differenz bestehen müßte.

Wir sind durch die Arbeiten auf babylonischem Gebiet berechtigt, ein hohes Alter für die Leberschaukunde, aber auch ein hohes Alter für ein ausgebildetes Leberschausystem anzunehmen (s. Jas trow, Relig. Babyl. u. Assyr. II. S. 273, auch für das Folgende). Das beweist die babylonische Tonleber, die als Schulmodell zur Unterweisung der Priester sicher aus der Hammurabi-Zeit, zirka 2000 vor Chr. stammt und bereits auf einem vollständig ausgebildeten System basiert. Auch in Boghaz-Köi, dem Mittelpunkte der hethitischen Kultur hat Winkler jüngst ähnliche Lebermodelle gefunden, die auch in ein hohes Alter hinaufreichen. Schon Gudea (ca. 2600 v. Chr.) erwähnt in seinen Inschriften die Leberschaukunde ${ }^{1}$ ) und zwar mit Ausdrücken, die auf ein Deutungssystem hinweisen. Besteht also, wie jetzt allgemein angenommen wird, zwischen dem bekannten babylonischen Lebermodell und der etruskischen Bronzeleber ein Zusammenhang, so sind wir berechtigt, letztere nicht als ein Neuerzeugnis des 3. Jahrhunderts vor Chr. zu betrachten, wo der ganze etruskischrömische Volksglaube seinem Verfall entgegenzugehen anfing, sondern als Kopie eines aus viel früherer Zeit stammenden Originals anzusehen. Auch von diesem Gesichtspunkte aus, weil eben die Leberschaukunde als System auf ein hohes Alter zurückgeht, sind wir berechtigt anzunehmen, daß durch die allmähliche Vertiefung in dieses System man zu allerlei, mehr oder minder phantastischen Spielereien (wie auch in der Astrologie) kam; aber auch $\mathrm{zu}$ mystischen oder kompliziert symbolischen Ausschreitungen.

Wenn ich also hier den Versuch mache, die Symbolik der Bronzeleber zu erklären, so möchte ich betonen, daß ich mir die Entwickelung dieser Symbolik als eine allmähliche vorstelle, die dadurch, daß sie von einer Priestergeneration zur andern übermittelt wurde, die Tendenz hatte, immer verwickelter zu werden.

Ich möchte noch auf eine andere Tatsache aufmerksam machen, welche meines Wissens in den bisherigen Bearbeitungen der Leber nicht erwähnt ist. Das ist die Verwendung eines Alphabets von 16 Buchstaben; also einer Anzahl, welche den 16 Buchstaben des

1) Die Stelle für Gudeas Erwähnung der Leberschaukunde ist Jastrow, Relig. Babyl. u. Assyr. II S. 273. Für die babylonische Tonleber und sonstige Zeugnisse für das Leberschausystem der Zeit des Hammurabi siehe Jastrow, c. c. 0 . II S. 218 u. 274 etc. Inzwischen sind noch zwei weitere Leberschautexte aus der Hammurabizeit von Dr. A. Ung n ad veröffentlicht worden. 
nordischen Runenalphabets sowohl, als der von den Alten vielfach angegebenen, von der modernen Forschung für unrichtige Überlieferung gehaltenen 16 -Zahl der Buchstaben für das älteste griechische Alphabet entspricht. Ganz abgesehen davon, daß in der Zahl der verwendeten Buchstaben die 16-Zahl der Randregionen und die für die etruskische Lehre verbürgte 16-Zahl der Himmelseinteilung (s. Thulin, Götter des Mart. Cap. S. 15 und Thulin, Etruskische Disziplin I S. 15) wiederkehrt ${ }^{1}$ ).

Das kann wohl kaum ein Zufall sein.

Bei der großen Sorgfalt, mit welcher die Stellung der Worte zueinander, die Richtung der Buchstaben, auf welche ich weiter unten, und S. 39 zurückkommen werde, die Kontrolle, welche die Worte von der Sonnenseite der Leber auf die Worte von der Nachtseite ausüben, die Größe der Regionen im Verhältnis zu ihrer Bedeutung abgewogen sind, ist man gezwungen anzunehmen, daß auch die Anzahl der Buchstaben absichtlich ist und ebenso einem hohen Alter des Alphabetes entspricht, wie die Worte, welche in eine Zeit weisen, zu welcher die persönlich gedachten Götter (bis auf den Sonnengottusil und Dionysos = fufluns) wenigstens für die Zwecke, die man mit dieser Leber verband, noch nicht vorhanden waren.

Wir müssen uns nicht wundern, wenn wir in der Anordnung der Linien und Worte auf der Leber auf eine ausgesprochen pedantische und, wenn man will, kindlich naive Genauigkeit stoßen. Wir haben in der Ausarbeitung des Horoskops bei den Astrologen des Mittelalters und in der Aufzeichnung von kabbalistischen Musterzeichnungen eine analoge Erscheinung, wobei man ebenfalls a uf die kleinsten Details hohen Wert legte und wobei dieselbe Spielerei mit Deutungen dieser Details stattfand, wie es, nach meiner Ansicht bei der etruskischen Bronzeleber der Fall ist.

Genauer ausgedrückt: Dieselbe Differenz, welche zwischen dem Inhalte und der Form der Bronze besteht, scheint auch zwischen der Form und der Anzahl der Buchstaben zu bestehen; Form der Bronze und Form der Buchstaben sind jung: Inhalt der Bronze und Anzahl der Buchstaben scheinen in ein ganz ausserordentlich hohes Alter hinaufzuweisen. Ich komme bei der Besprechung der einzelnen Worte immer wieder auf diese Erscheinung zurück.

Da Gestalt, Größe und Einteilung durch die Arbeiten von Deecke, Körte und Thulin endgültig festgestellt sind, so beginne ich sogleich mit dem, was mir die Anordnung der Linien und Worte, sowie deren Stellung $\mathrm{zu}$ einander $\mathrm{zu}$ sagen scheinen und verspare die Erklärung, wie ich dazu gekommen bin, für eine spätere Arbeit,

1) Ich werde in einem Nachtrag zur vorliegenden Arbeit auf die 16-Zahl näher zurückkommen. 
weil dazu hier der Raum mangelt und es sich jetzt nur um den Versuch handelt, die Leber in ihrer symbolischen Bedeutung zu erklären.

Wir müssen bei der Betrachtung der etruskischen (und babylonischen?) Lebern vor allem den Standpunkt festhalten, daß dieselben ein Abbild des Makrokosmos darstellen; daß sie die sichtbare Darstellung des sehr verwickelten göttlichen Systems und der Weltordnung sind; (Körte, Bronzeleber von Piacenza, S. 362) und daß diese Darstellungen mit großer Sorgfalt die vielfachen Bedeutungen so wiedergeben mußten, daß Irrtümer möglichst ausgeschlossen wurden, sobald sich die Leber-Späher bei der Leberschau danach zu richten hatten.

Man wird vielleicht annehmen müssen, daß Lebermodelle mit In- und Vorschriften erst gemacht werden mußten, als der Bedarf an Deutern größer war, als die Anzahl der geborenen Seher. Für solche also, die le rnen mußten, was die dazu geborenen konnten, wurden vielleicht Lebermodelle gemacht. Deshalb ist nicht nur jedes Wort an sich wichtig, welches auf der Leber steht, sondern es ist, wie ich schon sagte, die Richtung der Buchstaben, die Größe der einzelnen Abteilungen, die Stellung der Worte zu den sie zunächst umgebenden, ihre Verweisung in den Rand oder in die Mitte, die Kontrolle, die eines auf das andere dadurch ausübt, daß̧ es rechts oder links vom Todesstrome steht, auch die Erhöhungen und die Löcher aufs genaueste und peinlichste zu beachten. Wir müssen uns in die Lage der jungen Priester versetzen, um die Bedeutung der Leber in ihrem ganzen Umfange zu verstehen, denn zweifellos hat Jastrow recht, wenn er sagt (Sign and Name for Planet in Babylonian S. 147) "the famous bronze liver of Piacenza, prepared like the Babylonian clay model of a liver as an object lesson for instruction in the temple schools" ") und ich will in dem Folgenden versuchen, darzustellen, wie ich mir denke, daß die Belehrung der jungen Priester vor sich gegangen sei ${ }^{2}$ ).

Vorerst also mußte sich der Lernende mit dem Rücken gegen Norden, dem Gesicht gegen Süden stellen, Osten zur Linken, Westen zur Rechten haben ${ }^{3}$ ) und danach die Lernleber so in die linke

1) S. auch Thulin, Götter des Mart. Capella S. 15; die beiden erhaltenen Thonlebern sind nach Boissier "modèles anatomiques, qui facilitaient aux candidats à l'haruspicine l'étude de la dissection animale ... et correspondaient à nos tableaux scolaires".

2) Neben dem Lehrzweck stellt die Leber meines Erachtens zugleich die sichtbare Erinnerung an den Verfasser oder Veranlasser der Ideen ihres Inhaltes dar. Wie die Bundeslade Jahweh, die Hostie Christus vorstellt, so scheint die Leber fufluns, den „Haarigen“, eine durch einen ungeheueren Zeitraum von der Dionysos-Vorstellung getrennte, uralte Vorform desselben vorzustellen.

3) Uber Südorientierung mit Osten links s. Thulin, Etrusk. Disziplin S. 16, Deecke, Etruskische Forsch. IV, S. 84, Thulin, Götter des Mart. Cap. 
Hand nehmen, daß die gewölbte Oberfläche in seiner Handfläche ruhte. Siehe Thulin, Götter des Mart. Cap. S. 14: „Alabasterleber ... welche eine männliche Deckelfigur einer Urne des Volterraner Museums in der linken Hand hält"; und Jastrow Hepatoscopy and Astrology in Babyl. and Assyr. S. 675 Anm.: "Soudinos, a Chaldaean augur, who writing the words "victory of the king" backwards on the palm of his hand, pressed the smooth side of the liver of a sacrificial animal on $h$ is $h$ and and then held the liver with the significant words to the gaze of the army, who regarded it as a sign sent by the gods." (Siehe auch Jastrow Relig. Babyl. und Assyr. II S. 320.)

Dadurch, daß die Oberfläche der Leber, das Gewölbte, in der Handfläche ruhte, wurde diese Oberfläche unsichtbar und da alles, Worte, Stellung und sonstige Vorgänge auf und mit der Leber symbolisch zu deuten sind, (wie z. B. das Halten in der linken Hand einen Hinweis auf die linke d. i. glückliche Seite enthält), so bedeutet das soviel als: daß auch die Worte, die auf der Oberfläche der Leber geschrieben stehen, auf etwas Unsichtbares gehen und zwar auf etwas Unsichtbares, welches oben auf, oder über dem gewölbten Sichtbaren (d. i. der Himmel, das Gewölbe des Himmels) wohnt. Ich erinnere hier nochmals daran, daf wenn wir berechtigt sind, in der Leber den Reflex des Makrokosmus zu sehen, wir auch berechtigt sind, den nächsten Schritt zu machen: nämlich in den zwei Seiten der Leber das Abbild des sichtbaren und des unsichtbaren Teiles des Universums zu sehen. Von den beiden Worten, welche auf der gewölbten Oberfläche der Leber stehen, ist das Wort usil von zwei etrusk. Spiegeln her einwandfrei als Bezeichnung des Sonnengottes bekannt. (Fabr. Corp. Insc. Ital. No. 2097 und 2142.) Dadurch, daß die Leber usil, den Inhaber des Lichtes, unsichtbar und allein über dem gewölbten Himmel wohnend darstellt, kommen wir zu der Erkenntnis, daß die etruskische Leber einen einzigen Gott, den Inhaber des ewig gedachten Lichtes (s. S. 10) als Beherrscher des Raumes über dem gewölbten Himmel annimmt und keine andern "Götter" neben ihm. Dieses wird sich im Laufe der Untersuchung bestätigen.

Herodot stellt an die Spitze der von den Persern verehrten

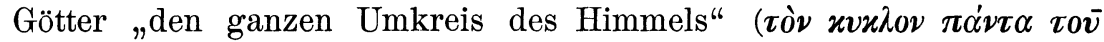
où $\alpha \nu o \bar{v}$ ). Jastrow, Relig. Babyl. u. Assyr. S. 227, Anm. 2 gibt das Wort sêru = „Rücken" für die gewölbte Oberfläche der babyl. Leber; beides stimmt mit usil von der Leber, das buchstäblich mit „Oben - Herr" zu übersetzen ist, wie ich glaube. Wenn wir berechtigt

S. 19 u. 20, Körte, Bronzeleber von Piacenza, S. 361 und Jastrow in der Zeitschrift für Assyriologie XXIII, S. 198-208 über die Orientierung bei der Astrologie in Babylon nach Süden. Über die Südorientierung bei den Chinesen s. de Groot, Religions System of China Vol. III Book I S. 984. 
sind, das babyl. Wort für die Oberfläche der Leber „sêru“, eigentlich "Rücken" mit siru = „hoch" in Verbindung zu setzen, so hätten wir auch bei der babyl. Leber eine Anspielung auf das "hohe“ oder obere Reich.

Weshalb das zweite Wort weder "Mond" bedeutet, wie bisher angenommen, noch die Alleinherrschaft des Sonnengottes beeinträchtigt, werde ich weiter unten erklären und hier nur bemerken, daßs es mir „die Helden“ zu bedeuten scheint, die mit dem ObenHerrn den Raum über dem gewölbten Himmel bewohnen; keine Götter, sondern zu himmlischen Bewohnern gemachte Sterbliche. (S. S. 16).

Auf der gewölbten Leberseite befinden sich mit usils (welches, wie ich glaube deshalb im Genitiv steht, weil der ganze Raum über dem Gewölbten des Oben-Herrn ist) auf der gleichen nördlichen Abteilung zwei Löcher; eines davon mehr gegen Norden, eines mehr westlich. Vielleicht entsprechen sie symbolisch dem winterlichen (mehr nördlichen) und dem sommerlichen (mehr westlichen) Untergangspunkte der Sonne. Das heißt: Sobald der Oben-Herr den gewölbten Himmel durch einen der beiden Ausgänge verläßt, ersehen wir dieses aus dem Versinken der Sonne, Winters gegen Norden, Sommers mehr in westlicher Richtung. Niemals aber ist der Herr, auch wenn er niedersteigt, vom gewölbten Himmel abwesend, sondern stets dort gegenwärtig gedacht, da man ihm kein Loch zum Hinauf- sondern nur eines zum Hinabsteigen gegeben hat.

Zur Erklärung sei hinzugefügt, daß bei der Bronzeleber, entsprechend ihrer Auffassung als Abbild des Weltalls, die Richtung der Worte, der Buchstaben und aller Vorgänge auf diesem Gerät entweder wie der Lauf der Himmelskörper von Osten nach Westen, oder mit der Richtung der etruskischen Schrift von rechts nach links, (letzteres besonders für die Randregionen) angenommen werden muß.

Zeichnet man die beiden Leberseiten, die gewölbte und die eng beschriebene auf durchsichtiges Papier und legt dann diese Zeichnungen genau aufeinander, so wird man finden, daß der nördlichere Ausgang Gottes (wir müssen von Gott, nicht von Göttern sprechen) derartig auf die Unterseite der Leber trifft, daß der äußere Rand des Loches den Götterberg (lobus pyramidalis oder caudatus) mit den Regionen 15 und $16^{1}$ ) verbindet. Das ist in der symbolischen Bedeutung der Leber: daß Gott sich zur winterlichen kühleren Zeit auf dem Götterberge niederläßst und daß er damit zugleich zu dem wird, was die erklärenden Inschriften, die er berührt, von ihm aussagen. Das ist in Reg. XVI tin $\boldsymbol{y} v f$ und in Reg. XV tin cilen.

1) Nach der Einteilung von Deecke und Thulin, die ich durchgehends beibehalten werde, da die Körtesche, die hier 8 und 7 hat, nicht zu stimmen scheint. 
Wie ich schon oben sagte, nicht tina oder tinia, der persönlich gestaltete Gott Juppiter wohnt in diesen Regionen, sondern der oberste Gott-Herr erscheint, indem er sich auf dem Götterberge niederläßt, als tin, also wie ich oben sagte: als im Amt des Juppiter. Ich will keine Übersetzung geben, weil das Mißtrauen, das ich damit erwecken würde, meiner Arbeit schaden könnte. Möge man sich also tin erklären, wie man wolle, so muß es doch eine auf Juppiter zu bezichende Sache sein und zwar eine, welche durch zwei hinzugefügte Worte nicht als dieselbe, sondern als zweierlei bezeichnet wird. Wir finden in Reg. XV tin durch cilen, in Reg. XVI tin durch $\vartheta v f$ näher bestimmt, welche beide Worte auf der Leber wiederkehren und zwar nimmt die dem Worte cilen ähnliche Form cilensl die größte Randregion der Leber ganz allein in Anspruch, während die Form des Randes XVI tin $9 v f$ in der Form tins $9 v f$ in einer Innenregion der rechten (nördlichen) Leberhälfte wiederkehrt, welche, wie mir scheint, zur näheren Bestimmung der Randregion II (das ist uni mae, welche sich unzweifelhaft auf Juno bezieht), vorhanden ist.

cilens erklärt Deecke Etrusk. Forsch. IV S. 50, 51 als Lua Saturni nach Varro; Thulin erklärt cilens als verhüllte Schicksalsgöttin und als identisch mit Favores opertanei (di involuti, superiores) des Martianus Capella ${ }^{1}$ ). Obgleich die Beweise bei Thulin schwach und wie Dr. Otto in seiner Besprechung in der dtsch. Literaturztg. vom 21. April 1909 No. 17 richtig sagt, vielfach unhaltbar sind und obgleich er cilens mit (wie mir scheint) der unrichtigen Region bei Mart. Cap. vergleicht, so erscheint es doch in diesem Falle, daß man für cilens seine Deutung als verhüllte Schicksalsgöttin oder besser noch als verhülltes Schicksal annehmen muß, und zwar, weil cilen mit der Wurzel hel = mors s. Grimm Gramm. II, S. 29 Nr. 314, Fick, Wörterb. II S. 83 urkeltisch kelô $=$ hehle, sanskr. kâla= Schwärze, urkelt. kelô-heben, sich regen, treiben, gehen zusammenfällt.

Doch es geht hier, wie bei tinia. In Reg. XV ist nicht von einer Göttin cilens die Rede, ebensowenig, als von dem Gotte tinia, sondern es stehen die Worte tin cilen als Erklärung (wie mir scheint) für das, was Gott wird oder was er tut, sobald er sich durch das Loch auf der Oberseite niederläßt und was also eine Mischung von juppiterlichen und verhüllten Funktionen sein muß.

Für $\vartheta v f$ haben wir gar keinen Anhalt, denn die Gründe sind nicht einleuchtend, welche verschiedene Etruskologen bewogen haben,

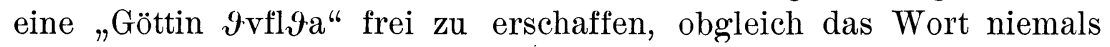

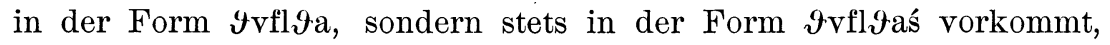

1) Auf die Ubereinstimmung der Regionen der Leber mit denen des Mart. Cap., welche, wie mir scheint, ohne die von Thulin für notwendig erachtete Verschiebung stattfindet, hoffe ich in einer besonderen Arbeit zurückzukommen. 
deren ś man teils als Genitiv, teils als Mehrzahl erklärt und einfach fortstreicht, um die Form $\vartheta v f l \vartheta a$ zu erhalten und für weiblich $\mathrm{zu}$ erklären, obgleich ja gerade der Name tina, tinia das a auch als Endung für einen männlichen Namen feststellt. Ebenso kommt $\vartheta v f l-$ Yaś auf keinem Spiegel als Göttin vor, ganz abgesehen davon, daß 9vflЭaś und 9vfl9icla (s. Thulin Götter des Mart. Cap. S. 34, 35, 36) unmöglich als dasselbe Wort in derselben Form wie $9 v f$ angesehen werden können.

Da wir also tatsächlich für eine Erklärung von $9 v f$ gar keinen Anhalt haben, so müssen wir, um eine Erklärung zu versuchen, uns an die Sagen halten, und zwar an solche Sagen, welche mit der Niederlassung des Sonnengottes auf dem Götterberge (wofür auch Deecke die Pyramide oder den processus caudatus hält, s. Etrusk. Forsch. IV, S. 10) zusammenhängen. Da finden wir denn jedesmal - ob wir Zeus-Juppiter nehmen, oder Odin-Wodan, oder den sinaitischen Berggott Jahweh, oder den babylonischen Enlil - diese obersten Himmelsinhaber, welche die Stelle des usil auf der Leber im Laufe der Zeiten okkupiert haben, übereinstimmend als Wetterwolken auf den Götterbergen lagernd; oder als Wetterwolken wandernd, sobald sie den himmlischen Saal verlassen; und zwar als drohende, Blitz, Hagel und Sturm in sich bergende Wolken.

Zeus = Jupiter=tinia, mit Aegis und Blitz sichtbar ausgestattet, sind also, wie mir scheint, "Gewordene" aus dem, was uns die Leber bildlich darstellt und durch die Inschriften des Randes in den Regionen XV und XVI erklärt: usil, der Sonnengott wird beim Niederlassen auf den Götterberg einmal tin cilen d. i. Vermittelung, oder Verwahrung, oder Verwaltung (des Lichtes) in Hülle oder in Verhüllung; zum andern Male tin 9vf, d. i.: Vermittelung, oder Verwahrung, oder Verwaltung (des Lichtes) in oder als Wetterwolke.

Für die Vorstellung des Zeus als himmlischen und unterirdischen, also als Wetterwolke oben auf dem Berge und als Verhüllten, wie

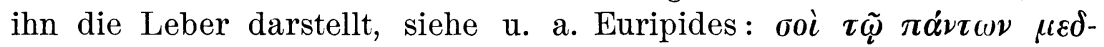

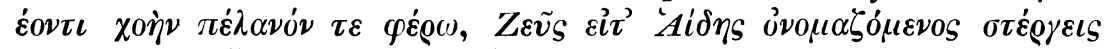

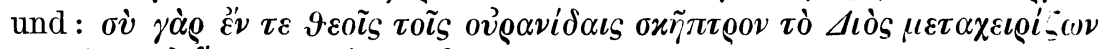

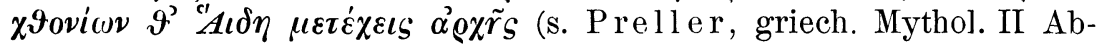
schnitt I Kap. Zeus. S. 105).

Auch stimmt die nähere Bestimmung der Juno in Reg. II durch die Jnnenregion als $\boldsymbol{y} \mathbf{v f}=$ Wetterwolke, da sie ja ebenso wie Zeus über Stürme, Nebel, Donner, Wolken und Blitz gebietet (s. Jlias $15,26 ; 21,6 ; 11,45 ; 8,433 ; 18,166,239)$.

Am Fuße des Götterberges finden wir zwei Inschriften, caףa und tins $\mathcal{\vartheta}$ ne $\boldsymbol{\vartheta}$, welche sich nur auf eben diesen Fuf des Götterberges beziehen können, da keine trennende Linie dazwischen steht.

Über die Wahrscheinlichkeit, daß ne $\mathcal{Y}$ die Wurzel oder das Element enthalten könnte, aus denen sich das etruskische Wort für 
Neptun, „ne 9 uns" später entwickelt hätte, habe ich schon S. 4 gesprochen und vorgeschlagen, ne $\vartheta$ mit „naß" zu erklären. Ich komme nun zu ca 9 a.

Nehmen wir tina, tinia, den Namen des persönlich gewordenen Jupiter als von tin abgeleitet, so müssen wir das, wegen der Ähnlichkeit der Form ebenfalls persönlich erscheinende cała von einem Stamm ca $\vartheta$ abgeleitet annehmen, der an sich so unpersönlich aufzufassen wäre, wie ne $\boldsymbol{Y}$ und tin und ebenso einen Zustand oder eine

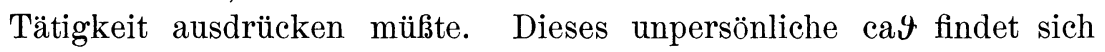
auf der Leber in der Randregion VI neben der Linie, welche die nördliche, oberirdisch gedachte Leberhälfte von der südlichen, unterirdisch gedachten scheidet und welche Linie, wie ich weiter unten ausführen werde, wohl mit dem Todesstrom identifiziert werden muß.

Wenn nun der, gleich Juppiter, persönliche "Gott" ca9a so eng mit dem Götterberge in Beziehung steht, wie es auf der Leber dargestellt ist, so also, daß cała gleichsam die Unterschrift des Götterberges bildet, also gleichsam der Götterberg selber ist und wir uns doch auf dem Götterberge nur einen einzigen Gott, den Sonnengott usil denken dürfen, so muß ca9a die etruskische Personifikation einer Sparte der Funktionen des Sonnengottes sein, wie Zeus und Juppiter griechische, resp. römische Personifikationen einer Sparte der Funktionen des Sonnengottes geworden sind ${ }^{\mathbf{1}}$ ).

Durch die Stellung des caЭa am Fuße des Götterberges ist weiter ausgedrückt, daß der "Gott" ca $\vartheta a$ erscheint, sobald usil, nach Verlassen des gewölbten Himmels am Fuße des Götterberges anlangt, also sobald er mit der Erde in Berührung kommt. Es scheint diese Stelle der Leber den Ursprung der unserer Zeit anstößig erscheinenden Verehrung der göttlichen Schöpferkraft zu erklären und zwar einmal als Hitze (Feuer), die dem Stein entstammt, sobald der Gott sich vom Himmel zur Erde herabläßt, einmal als Hitze (Feuer), die dem Holze entstammt, sobald der Gott aus der Unterwelt emporsteigt. Die auf der Leber vorhandenen Symbole dafür sind Götterberg(Donnerkeil) und Keule, die je dem lobus caudatus und der Gallenblase entsprechen. Es steht dieser Annahme nicht im Wege, daß ich mir den Ober-Herrn bei der symbolischen Erklärung als "stets im Himmel anwesend" vorstelle, wie S. 10 gesagt. Sein Herabsteigen zum Götterberg will nur eine Tätigkeit dieser Gottheit ausdrücken; ebenso muß man ihn als Unterirdischen auffassen, sowie in seinen andern, weiter unten zu besprechenden Gestalten, die immer nur Verkörperungen der verschiedenen Tätigkeiten Gottes - als Gewittergott, als Feuer vom Himmel zur Erde, oder aus dem Erdinnern zur Oberfläche sendend - darstellen.

1) Auch Thulin hält caףa für eine Form des Sonnengottes (s. Götter des Mart. Cap. S. 50), sowie Tor p, Etruscan Notes 1905, S. 9. 
Gerade diese Stelle der Leber mit der Darstellung des Gottes caya als Lebensspender scheint den Gedanken nahe zu legen, daß der ganze Inhalt der Leber sehr wahrscheinlich altasiatische Uberlieferung ist, die wegen ihrer Heiligkeit mit jenen Worten, die danach viel älter sein müssen, als das italienische Etruskisch des cippus perusinus und älter als das Etruskisch der Mumienbinden, immer wieder, von Jahrhundert zu Jahrhundert, unverändert auf neue Leberexemplare übertragen sind, lange nachdem sich der präzise umschriebene Sinn der Leberworte schon durch Zeit und räumliche Verschiebungen verwischt hatte. Siehe S. 36.

Ich komme nun zu dem zweiten der Löcher von der Oberseite der Leber, die ich als Ausgänge für usil, den Sonnengott ansehe. Der zweite heißere sommerlichere Ausgang Gottes trifft mitten in die Region 14 cilensl. Sie ist die größte der 16 Randregionen, deren sechsten Teil sie einnimmt und dadurch als die weiteste, ausgedehntestgedachte von allen bezeichnet. Da der Ausgang des Gottes mitten hineintrifft, so bedeutet das wohl, daß er selbst völlig inmitten dieser Region, der Region der "Verhüllten“ (auch nach Thulin, Götter des Mart. Cap. S. 36) versinkt. Da hier das große Reich der Verhüllten zu liegen scheint, so scheint das nach der Ausdrucksweise von der Leber auszusagen, daß an dieser Stelle die sämtlichen Verhüllten (di involuti) als zusammen wohnend gedacht sind. Um nun zu wissen, welche anderen Verhüllten in diese Region gehören, braucht man nur die Zahlen anzusehen. Die große Region der Verhüllten ist die 14.; die Zahl 14 enthält die Zahl 7 zweimal, folglich müssen wir das, was sich in denjenigen Regionen findet, die die Zahl 7 enthalten, als zwei andere Formen der Verhüllten in Anspruch nehmen.

Ich bin mir wohl bewußt, daß es willkürlich erscheinen kann, in dieser Weise eine Region der Leber mit einer anderen zu verbinden oder in Zusammenhang zu bringen. Aber die Zahl 7 ist als heilige und mystische Zahl so weit verbreitet unter den verschiedenen Völkern des Altertums, daß wir gewiß berechtigt sind, auch bei den Etruskern ähnliche Spielereien mit dieser Zahl zu finden, wie bei andern Völkern. Man vergleiche z. B. Hehn: Die Siebenzahl bei den Babyloniern in seinem Werk "Siebenzahl und Sabbat bei den Babyloniern und im alten Testament".

Zählen wir von Reg. 1, ani, nach links weiter, so trifft die erste 7 auf fufluns, welches Wort von den Spiegeln ganz unanzweifelbar als Bacchos = Dionysos bekannt ist.

Es steht damit nach meiner Auffassung wie mit tinia. So gut sich tinia aus dem Begriff der "Vermittlung des Lichtes" zu Jupiter verdichtet zu haben scheint, so gut hat sich vielleicht fufluns = Dionysus $=$ Bacchos aus dem Begriffe der wolligen, haarigen Verkörperung Gottes zum selbständigen Gotte verdichtet, denn die buchstäbliche 
Bedeutung der Randregion 7 fufluns scheint mir die des Wolligen, Haarigen.

Ich muß damit leider in das Gebiet der Etymologie hinübergreifen, selbst auf die Gefahr hin, meine Arbeit dadurch als verdächtig hinzustellen. Möglich, daß ich mit dem System, welches ich für die Worterklärung ausgearbeitet habe, auf einem falschen Wege bin, wie alle ohne Ausnahme, die sich bisher mit der Erklärung des Etruskischen beschäftigt haben. Ich möchte nur soviel sagen, daß die Resultate, die ich bisher erlangt habe, durchweg die alte Überlieferung mit der modernsten Forschung, soweit dieselbe unbezweifelbar feststeht, in Einklang setzt. Aber selbstverständlich kann erst die Zeit lehren, ob dieses System etwas taugt, oder nach kurzer Zeit versagt, wie alle andern Versuche und ich will deshalb, so viel ich kann, alles Etymologisieren bei der Deutung der Leber vermeiden, wie ich schon oben sagte. Doch ist es beinahe unmöglich, ein Dokument aus irgend einer Sprache zu deuten -- selbst wenn es so sorgsam und deutlich zusammengestellt ist, wie die Leber ohne auf die Worte der Sprache, um welche es sich handelt, einzugehen.

Ich komme also auf fufluns $=$ Dionysos $=$ Bacchos zurück, welches aus der Wurzel fufl, welche „wollig“ "haarig" „voll" zu bedeuten scheint, abgeleitet zu sein scheint, wie ne $\boldsymbol{\jmath}$ uns von ne $\boldsymbol{\vartheta}$, tinia von tin, ca 9 a ron cał und zwar so, daßs etrusk. f sich aus einem älteren vollen u entwickelt hätte und die Wurzel fufl dem bei Grimm Gramm. I S. 137 besprochenen uuul gleich stände; dazu Fick vailo-s $=$ Wolf u. valos mächtig, vla. Weil die Wortformen, die sich aus fufl entwickeln, zu den Namen für „Wolf" leiten, so scheint sich die Vorstellung, welche die Leber gibt, dahin aufzuklären, daß der Verhüllte in Tiergestalt, also als Wolf, zu denken sei, sobald er sich zu Reg. 7 in Beziehung setzt.

Diese Vorstellung zeigt ganz besonders das hohe Alter des Leberinhaltes (nicht der Bronze), denn sie weist in eine Zeit rückwärts, in welcher das Tier, welches späteren Geschlechtern als Begleiter oder Attribut der Götter galt, als eine direkte Verkleidung oder Hülle des obersten Gott-Herrn, des Sonnengottes aufgefaßit wurde.

Region 7 ist dadurch noch als ganz besonders bedeutungsvoll bezeichnet, daß das dritte Loch, welches sich auf der Oberseite der Leber befindet, hierher trifft und gerade diese Tatsache scheint die genaue Erklärung dessen $\mathrm{zu}$ enthalten, was man sich unter dem "andern Verhüllten“ dachte. Ich muß deshalb die große Region der Verhüllten (14) sowie die beiden dazu gehörigen Formen verlassen, um tivr zu erklären, weil dadurch das Verständnis erleichtert wird.

Das Wort tivr ist derart neben das Loch auf der Oberseite geschrieben, daß keine Linie dazwischen liegt, so daß es dadurch als 
dazu gehörig bezeichnet ist. Dann ist das Loch derart in die Bahn oder Linie, welche die Oberseite der Leber in zwei Hälften scheidet, hineingezeichnet, daß es ausdrücklich wie ein Eingang dazu aussieht. Weil wir außerdem allen Anfang in den Osten setzen müssen und allen Ausgang in den Westen (ganz einfach, weil die Laufbahn der Gestirne ebenfalls diesen Weg nimmt), so ist damit, daß tivr und das Loch im Osten der Bahn liegen, gesagt, daß hier ihre himmlische Bahn beginnt. Und weil dieses Loch oder dieser Eingang über Reg. 7 liegt, so heißt das, daß tivr aus Reg. 7 durch das Loch zum gewölbten Himmel, dem Wohnsitze des obersten GottHerrn emporsteigen.

Ich setze die bisherige Deutung für tivr, das einmal in der Form tivrs auf einem Sarkophage vorkommt (Fabr. C. J. 2119) (s. auch Deecke Etrusk. Forsch. IV. S. 8, der auf der Leber tivs liest und es mit "Mond“, „tivrs" mit „mensium" übersetzt) hierher; und zwar aus Skutsch, Etrusker, (Pauly-Wissowa Realenzyklopädie), - "Inschrift", — „wo nach dem Namen des Verstorbenen steht: avils XX tivrs śas. śa ist als Einerzahl von den Würfeln her bekannt. Für tivrs bleiben sonach nur zwei Möglichkeiten: entweder es ist verbindende Partikel, im Sinne von „und" - oder es heißt "Monate" bezw. "Tage". Man kann die erstere Deutung (als Partikel) ohne weiteres beiseite schieben, denn tivrs zeigt dieselbe Endung, wie avils und śas und es ist nicht wahrscheinlich, daß die Zahlenangabe bei avils aus Ziffer und Zahlwort gemischt sein sollte. Aber auch die Deutung "Tage" läßst sich mit Sicherheit ausschließen, sowie man sich an die Rückseite der Bronzeleber erinnert. Deren beide Hälften waren bezeichnet mit usils „Solis" und tivs, also "Lunae". Dadurch ist der Sinn "Monate" für tivrs sichergestellt."

Soweit Skuts ch. Daß diese Deutung nicht ohne weiteres einleuchtet, liegt auf der Hand, da man hier ein Wort, für welches man "und" oder "Monate" oder "Tage" ratet, dadurch "sicherstellt", daß man dasselbe Wort, nur weil es neben dem Namen des Sonnengottes steht, als "Mond" zum andern Male ebenfalls erratet. Selbst wenn der etruskische Name für "Mond" nicht sichergestellt sein würde, wie er es doch tatsächlich ist, nämlich bei Macrob. Sat. I, 15 „idus“" („itus“) etruskisch "Vollmond", auf das ich weiter unten, bei Besprechung des Viertelellipsoids (lobus papillaris) S. 25 zurückkommen werde, weil es sich durch die vollständige Übereinstimmung mit der babylonischen Auffassung, daß die Gestirne die „Sprecher" der Götter oder ihre "Schafe" sind (s. Jastrow, Name and Sign for Planet in.Babylonian S. 150) als ri chtig überliefert erweist; selbst wenn wir also den Namen für "Mond" nicht kennen würden, (was aber unbezweifelbar der Fall ist), so zeigt die Beweisführung über die Bedeutung von tivr so deutlich das beliebte Ratsystem, daß man nicht 
überzeugt wird und daß zum mindesten neue Deutungen eben so große Berechtigung haben.

Mir scheint tivr auf Grund eingehender Untersuchungen eine Pluralform zu sein, worin ich mit De ecke, Etrusk. Forsch. IV S. 8 übereinstimme, und scheint mir „Helden" zu heißen. Den Beweis dafür hoffe ich in meiner ausführlicheren Arbeit zu bringen, weil es sich hier nur um die Deutung der Leber handelt.

Es fragt sich nun, was die Bahn, der Weg, die Linie bedeutet, die so ganz speziell für tivr (das sind diejenigen, die aus der Region 7 des Wölfischen $=$ Dionysos $=$ fufluns zu Gott emporsteigen) vorhanden zu sein scheint. Wenn die gewölbte Oberseite der Leber als Symbol für den gewölbten Himmel anzunehmen ist, so muß die Linie, der Weg einer am gewölbten Himmel vorhandenen Linie oder Straße entsprechen und da es für diesen Begriff nur den Regenbogen, oder die Milchstraße gibt, so muß eines von beiden gemeint sein.

Ich glaube, daß man für die Leber den Regenbogen ausschließen kann, weil sein unregelmäßiges Auftreten ihn wohl als Weg für eine flüchtige Botschaft der Götter eignet, nicht aber als beständige Straße, die jeden Augenblick von den zu Gott emporsteigenden tivr benutzt werden kann.

Die Namen der Milchstraße weisen bei verschiedenen Völkern auf die Funktion hin, die die Leber ihr zuteilt, nämlich als "Weg".

Bei den Römern ist die nahe Verwandtschaft, in der die meisten ihrer religiösen Anschauungen zum Inhalte der Leber sowie zum Etruskischen üherhaupt stehen, auch durch den Namen der Milchstraße ausgedrückt; nämlich „Weg zu den Göttern", oder „Weg der Götter“ (s. Grimm, Mythol. S. 331, auch für die folgenden Bezeichnungen) ${ }^{1}$ ), arab. madscherra $=$ tractus, oder nahr al-madscherra $=$ flumen tractus, türkisch „Weg der Waller", welsch "Wodansweg“" etc. ${ }^{2}$ ).

1) Davon bezieht sich das römische „Weg der Götter" auf eine Zeit, in der die adoptierten Söhne Gottes „tivr“ bereits zu selbständigen Göttern geworden waren. Das Wort "Milchstraße" birgt vielleicht insofern Beziehung zum Leberinhalt, als wir in den Sagen von der den Herkules säugenden Hera vielleicht eine uralte symbolische Adoptionshandlung sehen dürften; quasi einen „Milchweg" in die Großfamilie. Auch Dionysos wird von Hera gesäugt (Eratosth. Catast. 44), also zur Aufnahme unter die Götter (= Weg zum Himmel) fühig gemacht. Man muß̉ auch an das heute noch geltende "Milchbruder " "Milchschwester" denken. Danach könnte das Milchopfer für die Toten als Erinnerung für die Götter an die "Milchverwandtschaft" gegolten haben. Siehe auch Ed. Meyer, Gesch. d. Altert. I, 2, S. 106 über die Säugung der egypt. Könige durch Göttinnen.

¿) Man könnte allerdings auch bei dem Wege am Himmel an die Ekliptik denken, die im Babylonischen als "Weg der Sonne" bezeichnet und in drei Teile geteilt wird, die als Wege für die uralte Dreiheit Anu, Enlil und Ea bezeichnet werden (s. Kugler, Sternkunde und Sterndienst in Babel I, S. 259). Aber nach meiner Ansicht entstammt die Symbolik der Leber einer Zeit, wo man noch nicht die Götter mit den Planeten identifizierte und einen fingierten Weg für sie feststellte. Deswegen ist hier als Weg eine Erscheinung am Himmel anzunehmen, die man tatsächlich sieht, also die Milchstraße. 
Auf die Leber übertragen: Gott, sobald er sich als ca9a manifestiert, taucht in der Region der Verhüllten, cilensl, unter; in dieser Region 14 der Verhüllten vermuten wir einen Hinweis auf die zweimal darin enthaltene Zahl 7 und die erste Reg. 7, auf welche wir treffen, ist die Reg. des Vollen, Wolligen, Haarigen, Wölfischen. fufluns = Dionysos, aus welcher die Helden (oder Halbgötter) zur Wohnung des Sonnengottes usil aufsteigen.

Um die Bedeutung der Reg. 7 ganz zu ermessen, müssen wir festhalten, zu wem sie am nächsten in Beziehung steht. Da finden wir in Reg. VI des Randes cay (= heiß oder hitzig), welches, da die trennende Linie zwischen ihm und der nächsten Innenregion "here“ (was ich mit „erdig“, „irdisch" übersetze) nicht vollständig durchgeführt ist, als in diese übergehend, oder mit derselben zusammenfließend gedacht ist.

Das bedeutet, nach Art der Darstellung auf der Leber, daß ca $\vartheta$ und herc sich hier vereinigen und zwar ist der Platz, wo dieses geschieht genau bezeichnet: zwischen der Keule, nämlich der Gallenblase und dem Beginne einer Linie, die, wie ich später (S. 20) ausführen werde, wahrscheinlich das nördliche Ufer des Todesstromes darstellt, mit dem ja bekanntlich die verschiedenen Völker allerlei Vorstellungen verknüpften.

Der Wölfische, der Erste der Verhüllten, fufluns = Dionysos erscheint dadurch, daß er auf dem Wege von rechts nach links der Nächste und das Nächste ist, worauf wir nach Reg. VI treffen, als aus dieser Vereinigung von ca 9 (göttliche Hitze) und herc (Kraft der Erde) hervorgegangen und danach göttlich und irdisch zugleich gedacht zu sein. In die Sprache der Leber übertragen: aus der Vereinigung des Gottes mit der Erde gehen wollige haarige Verhüllungen, (der Gott-Wolf-Dionysos-Apollo, vielleicht der Gott-Stier-Dionysos-Bacchos, der Gott-Löwe-Herkules hervor), Halbgötter und Stammesgötter, denen dann das eigentlich menschliche Geschlecht erst nachfolgt.

Es erklärt hier die Leber eine solche Menge von erhaltenen Sagen und Gebräuchen, daß man aus der Fülle nur auf ganz Einzelnes hinweisen kann. Das Wichtigste, was uns für diese Darstellung von der Leber erhalten ist, scheint die Bezeichnung der die verschiedenen Wanderungszüge der Völker leitenden Heerführer als "Wölfe". Wie aus der Region des Wölfischen (fufluns) die Helden (tivr) in das Land der ewigen Sonne (usil) gehen, so führt der menschliche "Wolf" (= Heerführer) zu irdischem Vorteil (s. Festus Pauli, ed. O. Müller S. 106, Paul. Diaconus, Hist. Longob. IV, 39 u. Strabo 2, 208). Für die Bezeichnung der Oberägyptischen Krieger als "Wolfsgestalten" und über den „Wolfsgott" s. Eduard Meyer, Geschichte des Altertums, I. 2. S. 49 u. $72^{1}$ ).

1) Vielleicht liegt in der Bezeichnung "haarig“, „wollig“ die Erinnerung an einen behaarten Vorfahren des heutigen Menschen aufbewahrt. Sie findet 
Thulin (Götter des Mart. Cap. S. 37, 38, 39) hat deshalb recht, wenn er aus dem Pales secundanus einen Favor Iovis filius primogenius herausliest (S. 38). Man muß nur für Iovis nicht den jüngern Juppiter, sondern den uralten Sonnengott usil in seiner dritten Emanation als "Hitze" caগa setzen.

Sehr wahrscheinlich enthält Reg. VII, fufluns, noch zwei andere symbolische Bedeutungen, welche lediglich durch die Zeichnung ausgedrückt sind. Daß es nicht Zufall ist, zeigt der Vergleich mit der anatomischen Leber. Es befindet sich in Reg. VII, genau da, wo diese an Reg. VI anstößt, eine Erhöhung und der Rand der Bronzeleber ist hier, ganz der anatomischen Leber entsprechend, vorgewölbt, zeichnet also diese Stelle als abweichend von der glatt verlaufenden Randlinie aus, ohne sie davon abzutrennen.

Die 16 Randregionen der Leber umschließen dieselbe auf einem bandartigen Streifen, der seiner Form nach wohl nur eine Schlange ausdrücken kann, als deren Haupt und Anfang die erhöhte Stelle in Reg. $7 \mathrm{zu}$ gelten hat. Was ich S. 18 über die Geburt des Wölfischen fufluns aus ca $\vartheta$ und herc gesagt habe, muß auch für die Schlange gelten, die aus dem gleichen Platz wie fufluns ihren Anfang nimmt. Auch sie muß von ca $\vartheta$ und herc geboren sein. Daß sie nicht als eine aalähnliche Schlange, sondern als eines jener uns fabelhaften Tiere aus einer früheren Erdperiode, also als eine Art von Hydro- oder Notho- oder Ichtyo-Saurus gedacht ist, zeigt das Wort, das sich auf ihrem Haupte (Reg. 7) befindet, welches zugleich fufluns ist. In der Sprache der Leber müßte das soviel heißen, als daß einerseits das Haupt der Schlange als ein haariges Tierhaupt gedacht ist, andererseits daß3 eben der Wollige, Haarige, fufluns $=$ Dionysos ihr Haupt ist, d. i. also stärker als die Schlange, obgleich an sich nur ein Teil, wenn auch der hauptsächlichste, von ihr; beide aber sind Kinder des obersten Gott-Herrn usil, aus seiner dritten Verkörperung als ca9a.

Buchstäblich enthält Saemundar-Edda 55a (s. Grim m, Mythol.S.754) diese Stelle der Leber und weil der Inhalt der etruskischen Leber ganz

sich im Babylonischen, wo Eabani, der Begleiter des Gilgameš, auf den Siegelzylindern als Urmensch haarig dargestellt ist. Vielleicht liegt auch in der Beschreibung des Esau als „haarig“ der Überrest einer primitiven Volksanschauung ähnlicher Art vor, um so mehr, als gewisse Züge bei Esau darauf hinweisen, daß - wie z. B. die Gleichsetzung mit Edom-Adam - Traditionen aus dem Volksglauben über den Urmenschen mit in die Sage aufgenommen wurden. Wegen der Beschreibung des Eabani s. Jensen, Keilinschriftliche Bibliothek VI, 1, S. 121: „Mit Haar bedeckt ist sein ganzer Leib, sein Haupthaar ist wie das eines Weibes, die Fülle seines Haupthaares reckt sich wie Weizen. Nicht kennt er Land und Leute, bekleidet ist er wie Gira. Mit den Gazellen zusammen frift er Kraut, mit dem Vieh zusammen sättigt er sich an der Tränke." S. auch Schneider, Entwickelung des Gilgameschepos, (Leipziger Semitische Studien V, 1, S. 55, 56), der zu dem Ausdruck „bekleidet wie Gira“ hinzufügt: „d. h. wohl mit Fellen". 
unverkennbar die gemeinsame uralte Quelle für die griechischen, römischen und germanischen Göttersagen darstellt, wie auch für die babylonischen und chinesischen Vorstellungen, wie ich es später zu zeigen hoffe, so setze ich die Stelle mit den Worten Jak o b Grim m s hierher.

„Nach der Edda windet sich eine ungeheure Schlange, der midgards ormr um den Erdkreis; offenbar das Weltmeer." — „Jene allen Göttern verhaßte ${ }^{1}$ ) Schlange war das Kind des Loki ${ }^{2}$ ), Bruder des Fenrisulfr ${ }^{3}$ ) und der $\left.\mathrm{Hel}^{4}\right)^{\text {". }}$.

Um nicht weitläufig zu werden, verzichte ich, hier auf die Anführung analoger Vorstellungen in bezug auf die Schlange bei andern Völkern einzugehen. Es genüge hier auf die betreffenden Artikel in verschiedenen Realenzyklopädien über das Altertum hinzuweisen, wie auch auf das Werk von Schwarz, Geschichte der Schlangengottheiten. Berlin 1859, Gruppe: Mythologie und Kulte der Griechen, Index sub Schlange.

Sehr wahrscheinlich ist auch mit der alles Irdische und Unterirdische umgürtenden Schlange ${ }^{5}$ ) der Leber das Weltmeer gemeint; das Weltmeer selber aber wieder als Symbol der Ewigkeit aufgefaßt, weil auf der Leber der Todesstrom, Reg. 17 le 9 am hineinmündet. Symbolisch: so gut der Strom sich mit dem Meere mischt, so mischt der Tote selber sich erst mit dem Vergessen, Lethe, und schließlich mit der unendlichen Zeit, d. i. die Ewigkeit.

Ich glaube, daß man den ganzen Raum, welchen die Reg. 17 einnimmt, als Todesstrom auffassen muß3 und daß die beiden Linien, welche das Wort le 9 am einschließen, als das nördliche und südliche Ufer des Todesstromes zu deuten sind. Von diesen beiden Ufern des Todesstromes ist das nördliche das wichtigere, ja wohl eine der wichtigsten Linien überhaupt, weil sie sehr wahrscheinlich eine Haupt-

1) Apollo mußste erst den Python töten, ehe er in den Besitz des Orakels zu Delphi gelangen konnte.

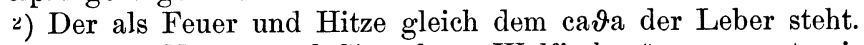

3) Der als Namen und Sinn dem "Wölfischen" genau entspricht.

4) D. i. cilen und entspricht genau in Stellung und Sinn der cilen $(=$ Hülle) in Reg. $7^{1}$, die auch auf der Leber als Kind von ea $\vartheta$ und herc gezeichnet ist. S. S. 31 .

5) Ich kann es nicht unterlassen, hierbei auf eine Darstellung an der Spitze einer babylonischen Inschrift des Königs Nabupaliddin (s. Bezold, Ninive und Babylon, S. 87) aufmerksam zu machen, auf der der Sonnengott dargestellt wird, auf einem Thron über dem Weltmeere (Apsu) sitzend und umrahmt von einer Schlange, de gewissermatien aus dem Weltmeer (das auch als unterirdischer Raum gedacht ist) aufsteigt. Auch kann ich nicht umhin, hier darauf aufmerksam zu machen, daß der Leberinhalt, so wie ich ihn gedeutet habe, buchstäblich der Bedeutung der $8 \mathrm{kwa}$ für die älteste chinesische Religion entspricht. Siehe de Groot, Religious-System of China, Vol. III, Book I, S. 959 sq. Diese Übereinstimmung ist so stark, daf ich in einem Nachtrage zu dieser vorliegenden Arbeit darauf ausführlicher zurückkommen mußj. 
ursache ist, weshalb die Alten die Leber und nicht das Herz als Sitz des Lebens ansahen.

Es entspricht nämlich diese Linie dem Einschnitt, welcher auf der anatomischen Leber durch das Nabelband (ligamentum teres) und ein Blutgefäß (ductus venosus Arantii) verursacht wird, welches letztere fötal das Blut vom Nabel zum Herzen führt. Die erste Station auf diesem Wege ist die Leber ${ }^{1}$ ), welche vor der Geburt unverhältnismäßig groß ist, so daß sie alle andern Organe des Unterleibes verdeckt; und dazu ist sie von dunkelroter Farbe. Eben daraus schloß man wohl, daß hier der Sitz des Lebens sei, während das kleinere Herz unbeachtet blieb (s. Körte, Bronzeleber von Piacenza S. 360).

Diesen Blutstrom, der vom Nabel zum Sitz des Lebens geht, zeichnet die Leber, wie ich glaube, in Reg. 17. Wenn ich damit recht habe, so muß der Ausgangspunkt des Stromes, also die erhöhte Stelle der Leber, die zugleich der Kopf der Schlange ist, genau wie es der Wirklichkeit entspricht, auch als Nabel gedacht sein, weil die Leber wirkliche mit symbolischen Vorstellungen vereinigt. Wir müssen also sehr wahrscheinlich den Kopf der Schlange oder besser gesagt ihren Rachen, zugleich als Omphalos, (der dem etruskischen mundus, dem deutschen Dillestein entspricht), also als Weltnabel und Eingang zur Unterwelt auffassen, von welchem, genau wie es der Wirklichkeit entspricht, der Blutstrom ausgeht, der, ebenfalls der Wirklichkeit entsprechend, die Leber in zwei Hälften teilt.

Wenn ich damit recht habe, so müßten die ältesten Vorstellungen des Todesstromes diesen als einen Strom von Blut und nicht von Wasser darstellen und zwar als einen Strom von Blut, welcher als Trank, d. i. zur Ernährung bestimmt war. Ja, es scheint sogar, als ob die Leber genau festsetzt, welcherlei Blut hier gemeint sei: nämlich das der Opfer.

Die Begrenzung des nördlichen Ufers des Todesstromes am Beginne seines Ursprungs im Osten wird von Reg. 19 gebildet, nämlich von herc und der damit zusammenfließenden Reg. 6, nämlich ca`, die beide auf der lichten, oberirdisch gedachten nördlichen Leberseite liegen. Das nördliche Ufer des Todesstromes vereinigt sich nirgends mit dem südlichen, sondern der Übergang aus dem Licht, oder Norden in die Finsternis, oder Süden, wird rom nördlichen zum südlichen Ufer des Todesstromes durch eine kleine Linie vermittelt, welche kaum etwas anderes sein kann, als eine Brücke. Unwillkürlich drängt sich hier der Gedanke an die Pontifices auf und es wäre nicht unwahrscheinlich, daß sie, indem sie ursprünglich das Blut der Opfer in strömendes Wasser rinnen ließen, die „Brücken-

1) Siehe Jastrow, Relig. Babyl. u. Assyr. II S. 215, Anm. 4: „Die Leber wird auch als "Mutter" des Herzens betrachtet"; und ebendaselbst S. 217: „Leber ist Quelle des Lebens" usw. Auch Körte weist auf den ductus venosus hin. 
macher" für den Übergang der Toten vom Licht ins Dunkle vorstellten und daß erst mit der Umwandlung des blutigen in ein unblutiges Opfer sie die Vorsteher unblutiger Opfer geworden seien. (Wegen der Brücken zur Unterwelt s. Grimm, Mythol. S. 794). Daß3 Reg. 17 leYam der „Fluß" der Leber ist, von dem Hesychius

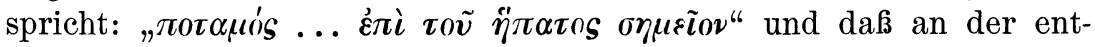
sprechenden Stelle auf den babylonischen Lebern ebenfalls der "Fluß" $\mathrm{zu}$ suchen ist, der in den Texten vorkommt, scheint einleuchtend. (S. Thulin, Etrusk. Disziplin II S. 54.)

Soeben erhalte ich die Mitteilung durch Prof. Jastrow, daß sich auf der babyl. Tonleber im Britischen Museum auf seine Anfrage hin zwei Paar der zahlreichen in die Leber eingedrückten Löcher durch je eine Rinne unter der Oberfläche des Tons verbunden zeigen. Diese Rinnen befinden sich an derjenigen Stelle, an welcher nach meiner Angabe der „Fluß" gesucht werden mußte, so daß auch hier volle Übereinstimmung waltet. Ja mehr: man hätte diese Rinnen sehr wahrscheinlich kaum beachtet, da sie unter der Oberfäche des Tonmodells verborgen liegen, wenn nicht auf der etruskischen Leber die Ufer des Todesstromes so deutlich gezeichnet wären.

Die breite Mündung des Todesstromes trifft auf die Schlange, wie ich schon S. 20 sagte; im Sinne von „Fluß" ins „Meer"; im Sinne von "Vergessenheit" in die „Ewigkeit". Die ganze Region, welche die Mündung des Todesstromes begrenzt (Reg. 13) ist mit vetisl bezeichnet.

Deecke und Thulin nehmen an, daß aus dem etruskischen Worte vetisl der Göttername Vedius = Veiovis entstanden sei und Thulin, der die Reihe der Götter des Martianus Capella um zwei Plätze verschieben muß, um eine Übereinstimmung mit der Leber zu erzielen, setzt den in Reg. XV bei Martian stehenden Veiovis mit dem in Reg. 13 der Leber stehenden vetisl gleich.

Ich glaube, daß die Götter des Martianus genau mit den Regionen der Leber, ohne Verschiebung übereinstimmen und daß man deshalb die Bezeichnung „Fata alii di Manium" in Reg. 13 bei Martian quasi als Erklärung für vetisl in Reg. 13 der Leber nehmen kann. Trotzdem haben Deecke und Thulin recht, wenn sie Vedius = Veiovis als aus vetisl entsprungen annehmen, nur ist, wie tinia aus tin, ne $\boldsymbol{y}$ uns aus ne $\boldsymbol{\vartheta}$, hercle aus herc so auch der lateinische Vedius aus der gleichen Wurzel mit etruskisch vet entsprungen. Wäre Etymologie erlaubt, so würde ich vorschlagen vetisl mit „Reich der Wissenden" zu übersetzen.

Die Zeichnung drückt aus: daß vetisl etwas ist, das unterirdisch, (weil jenseits des abgrenzenden Ufers des Todesstromes) wohnt und zwar nur dort, wo der Blutstrom ins Meer mündet; daß es in nächster Nachbarschaft zur Zunge Gottes steht, aber nicht die Zunge selber ist. $\quad$ (Zunge $=$ lobus oder processus papillaris, der, wie ich S. 24 
ausführen werde, als symbolische Zunge und zwar Zunge Gottes zu deuten ist, im Sinne von "Sprecher(); daß vetisl in der 7. Region der Unterwelt wohnt: daß es im ganzen genommen das Schlimmste von der Leber überhaupt ist, da es die rein westliche, in der Mündung des Blutstromes gelegene 13. Region einnimmt. Wegen der Bedeutung der Himmelsgegenden als glücklich und unglücklich s. u. a. Thulin, Etruskische Disziplin I, S. 18; und wegen der Bedeutung der Zahl 13: besteht nicht noch heute der Glaube, daß derjenige, der als 13ter an der Tafel (des Schicksals) sitzt, dem Tode (d. i. der Mündung des Todesstromes vetisl) geweiht ist?

Der Sinn der Zeichnung von der Leber liegt, wie es scheint, unter anderen Sagen auch in der Erzählung vom Odysseus, wie er die Seelen aus dem Hades beschwört: sobald die Seelen der Verstorbenen vom Opferblute getrunken haben, werden sie fähig sich gewissen Lebenden verständlich $\mathrm{zu}$ machen; d. i. in der Sprache der Leber: die Wissenden, die vom Opferstrome genährt sind, reden zu gewissen Lebenden, d. h. sie machen sie wissend, wie sie selber. Ich erinnere hier nur an Bigois, die Verfasserin der Blitzlehre (s. Thulin, Etruskische Disziplin S. 4, 5, 6, 7), die Thulin unzweifelhaft richtig mit Vegoia gleichstellt (s. auch K ö rt e, Bronzeleber von Piacenza S. 358) und deren Namensformen merkwürdig an Veiovis = Vedius gemahnen; auch sie "machte wissend" 1 ).

Ich möchte hier noch die Vermutung aussprechen, daß die Fissa, die Thulin in seiner etruskischen Disziplin II. S. 40 bespricht, sich wahrscheinlich auf die Linien beziehen, welche den Todesstrom bilden und zwar derart, daß das Fissum familiare sich auf das nördliche, das Fissum vitale sich auf das südliche Ufer des Todesstromes bezieht. Wenn die Keilschrifttexte von drei Einschnitten sprechen, (Thulin, Etruskische Disziplin II, 41), so wäre es möglich, daß mit dem dritten Fissum die „Brücke“ gemeint wäre, oder, genauer ausgedrückt, der Raum unterhalb der Brücke. Aber da, wie mir mitgeteilt wird, die Anzahl der Spalten in den babylonischen Leberschautexten nicht auf 3 beschränkt ist, sondern bis auf 7 geht, so ist hierauf kein besonderer Wert $\mathrm{zu}$ legen. Im allgemeinen sei bemerkt, daß es scheint, als ob die babylonische Leberschau sich aus den Grundlagen, wie sie die etruskische Leber aus einer viel früheren Epoche festgehalten hat, selbständig weiter entwickelte; während die römischen Überlieferungen ein genaues Festhalten an den etruskischen Vorschriften bekunden; doch so, als ob die etruskischen Kultuslehrer die innerste mystische Bedeutung den römischen Kultusschülern vorenthalten hätten. Es scheint in andern Worten, als ob die Römer,

1) Dieses bezieht sich auf den Sinn des Namens der Bigois. Etymologisch hat G. Herbig unzweifelhaft recht, wenn er Begoe bei Servius als latinisiertes etruskisches vecui ansieht. 
weil ihnen die Kenntnis der Ursachen der Vorschriften fehlte, furchtsam an eben diesen Vorschriften festhielten, während die Babylonier, bei voller Kenntnis der Ursachen der Vorschriften, diese je nach Bedürfnis erweitern und beweglich machen konnten.

Wenn die erste 7te Region, welche in der "Verhüllten" 14 enthalten ist, dahin $\mathrm{zu}$ deuten ist, daß Söhne Gottes in derselben wohnen, so muß dasselbe bei der zweiten 7 ten Region der Fall sein; und wenn die Deutung richtig ist, daß in der ersten 7 der wölfische, wollige haarige Sohn Gottes wohnt, und aus dieser Region die wölfischen, heldischen Söhne Gottes zu Gott emporsteigen, um mit ihm als Halbgötter und Stammgötter über dem gewölbten Himmel zu wohnen, so müßte man die Deutung dafür, welcherlei Art vetisl sei, ebenfalls aus der Stellung der Region 13 erschließen können.

Da wir, der Schriftrichtung gleich, von rechts nach links am Leberrand entlang gehen müssen, so kommt vetisl (13) aus cvlalp (12) und grenzt an cilensl (14). Für cvlalp fehlt uns jeder Anhaltspunkt (s. die sich widersprechenden Deutungen bei Thulin, Götter des Mart. Cap. S. 41 von cvl und alp). Wenn Etymologie erlaubt wäre, so würde ich vorschlagen cvlalp mit "Krankheit" zu übersetzen; die Region „Krankheit" als Vorstufe zur Region Fata alii di Manium zu nehmen, wäre nicht ganz sinnlos.

Daß3 das Viertelellipsoid, welches dem Processus papillaris der anatomischen Leber entspricht, die nächste Nachbarschaft bildet, sagte ich schon. Da das Viertelellipsoid, wie ich weiter unten sogleich versuchen werde, zu zeigen, als "Zunge" oder "Sprecher" Gottes anzusehen ist, so sind die der Zunge, (also dem Vermittler des göttlichen Willens) so nahe Stehenden gleichsam als die Vertreter der geistigen Kräfte Gottes anzusehen, wie die Wölfischen der körperlichen Kräfte Gottes. Vetisl muß3 also die Region der dem Vermittler des göttlichen Willens Zunächststehenden, das sind zweifellos die Weissager, Priester, Verkünder, Gesetze-Singer, kurz (auch ohne Etymologie) die „Wisser", wofür ja auch die indogermanische Wortwurzei Anhaltspunkte bietet.

Dadurch, daß ihre Wohnung im Westen liegt, ist ihre Macht als "fürchterlicher" bezeichnet, als die der „Wölfischen"; dadurch, daß̂ sie erst in der zweiten Verhüllung auftreten, ist ihre Stellung, als um ein geringes weiter ab vom obersten Gott, sie selber also als nicht ganz so alt, als die Wölfischen bestimmt; ganz wie es der Wirklichkeit, in welcher Kraft-Herrschaft vor Geistes-Herrschaft kommt, entspricht. Da die am gewölbten Himmel gezeichnete Straße „der Weg zu den Göttern" (Milchstraße) im Osten aus dem Meere bei dem Wölfischen aufsteigt, im Westen bei den Wissenden ins Meer niedergeht, so sind sie auch durch dieses Band als verbunden und als Stationen ein er Straße dargestellt.

Ich komme nun zu dem Viertelellipsoid. 
Genau wie in Wirklichkeit der Ductus venosus, also die Zufuhr des Blutstromes, vom Nabel zur Leber geht, genau so geht das nördliche Ufer des Todesstromes vom Omphalos auf das Viertelellipsoid zu und wenn ein symbolischer Nabel vorhanden ist, so muß auch eine symbolische Leber vorhanden sein; und ebenso: wie der symbolische Nabel den Eingang in den Bauch der Frde darstellt, so muß die symbolische Leber den Sitz des Lebens im Makrokosmus bedeuten, wie die wirkliche beim einzelnen Individuum. Weil nun die Leber der Opfertiere als das direkte Mittel zur Überlieferung des Willens der Götter an die Menschen galt und zwar als das irdisch greifbare (s. Jastrow, Sign and Name for Planet in Babylonian S. 145: "the liver of the animal reflects the mind and will of the god", und Thulin, Etruskische Disziplin II, sowie Jastrow, Relig. Babyl. u. Assyr. II S. 212, 213), so muß diese wiederum andererseits das Abbild oder Symbol für ein himmlisch gedachtes Wahrzeichen für "mind and will of the god" sein; beide zusammen aber müssen, da sie für den Gott reden, oder an Stelle Gottes reden, als seine "Zunge" gedacht sein. (Über die in der griechischen und babylonischen Leberschau vorkommende "Zunge“, s. J a strow, Relig. Babyl. u. Assyr. II S. 325).

Man könnte hiergegen einwenden, daß ich nicht berechtigt sei, bei meinem Versuch die Leber $\mathrm{zu}$ deuten, einen bestimmten Teil und dazu noch einen, der nicht zu den hervorragenden Erscheinungen auf der Leber gehört, mit einer so allgemeinen Anschauung wie "Zunge" zu verknüpfen - eine Deutung, die sich eigentlich auf die Leber als Ganzes gedacht, bezieht. Dem ist entgegenzuhalten, daß nach der hier vertretenen Auffassung die Leber das Abbild des Weltganzen darstellt und jeder einzelne Teil in Verbindung mit einer bestimmten Tätigkeit des Weltgottes zu bringen ist. Und wenn ich recht habe, daß man bei der Anfertigung dieses heiligen Gerätes auf jede Einzelheit Wert legte, so erscheint die Methode gerechtfertigt, auch bei einem (auf der anatomischen Leber) geringeren Leberbestandteil, der aber bei der Darstellung deutlich hervorgehoben ist, den Reflex einer wichtigen göttlichen Tätigkeit zu erkennen.

Was unter der himmlischen Zunge Gottes, deren irdisches Symbol die ${ }_{n}$ sprechende" Opferleber ist, gedacht war, ergibt sich sehr klar aus der Ubereinstimmung der Worte: assyr. Lu-Bat für "Schaf" und „Planet", s. Jastrow, Sign and Name for Planet in Babylonian, S. 151 und Eisler, the Origin of Eucharist (Beilage der Münchner Neuesten Nachrichten, Nr. 96, S. 183), der das gemeinsemitische Wort "Amar" für "Schaf" und "Wort" bespricht. Das ist also : so gut aus dem irdischen Schafe die Leber als Zunge Gottes spricht, so gut gelten auch die himmlischen Schafe, das sind die Planeten, als Sprecher Gottes.

Aus der Leber geht hervor, daß nicht eine Mehrzahl von Planeten, (Schafen oder Zungen), sondern eine eỉnzige himmlische 
Zunge anzunehmen ist; einmal, weil soweit ich sehe, nur eine Zunge dargestellt ist und zum andern Mal, weil die Unterschrift, welche das Viertelellipsoid erklären soll (von Körte in seiner Reg. 34 als Эetlvm $\vartheta$ richtig, von Deecke $13^{\prime} \boldsymbol{Y e t l v m r}$ falsch gelesen), in der Einzahl steht. Die Bedeutung von $\bigoplus$ etlvm $\vartheta$ scheint mir buchstäblich "der die Zeit erleuchtet" zu sein, was sowohl auf ein periodisch leuchtendes Himmelsschaf, als auf ein die Entfernung der Zeit ermessendes oder sie erleuchtendes sich beziehen kann. Da die Deutung Deeckes (Etrusk. Forsch. IV. S. 41, 42), die er von $\vartheta$ etlvmr gibt, dadurch hinfällig geworden ist, daß das Wort te $\boldsymbol{\xi} v \mathrm{vm}$, worauf er sie stützt, nicht existiert, sondern sich als tecvm herausgestellt hat und Thulin überhaupt keine Deutung des Wortes Jet$\operatorname{lvm} \vartheta$ gibt, so darf man vielleicht, selbst wenn meine Deutung nur teilweise richtig wäre, in dieser Weise vom Unsichern zum Sichern eine Brücke schlagen.

In Frage käme von den Planeten in erster Linie Merkur „der auch aus besonderen Gründen, als der Planet par excellence" galt, wie Jastrow ihn nennt, (s. Sign and Name for Planet in Babyl. S. 152, 153) weil er "sharply separated from his fellows ist", weil er allein "double sexed" ist, weil "qualities are heaped upon Mercury in profusion", weil er die "soul among the planets" ist, weil er der "Planet of the language" ist, weil er "assigned to the liver, as the organ of revelation" ist.

Trotzdem glaube ich, daß das Viertelellipsoid der Leber nicht für Merkur, sondern für den Mond steht und daßs der Begriff des Merkur, als Gott und als Planet sich viel später für einen Teil der Funktionen des Mondes, nämlich für das Wandern, Sprechen und den heilenden Einfluß des Mondlichtes selbstständig entwickelt hat ${ }^{1}$ ).

Erstens sagt Jastrow (Sign and Name for Planet in Babyl. S. 153), daß die sämtlichen oben angeführten reichen Eigenschaften des Planeten Merkur der griechischen Astrologie angehören ${ }^{2}$ ), und daß diese "associations of ideas have not as yet been encountered in Babylonian texts", und zweitens gibt die etruskische Sprache für "Schaf" nicht „Planet", wie im Babylonischen, sondern zweifellos "Mond". Nämlich Deecke Etrusk. Forsch. IV S. 8 "idus"

1) Professor Jastrow teilt mir mit, dab er jetzt auf Grund weiterer Studien astrologischer Texte bestätigen kann, daß Merkur gewissermaßen das Residuum unter den Planeten darstellt, also zunächst der unbedeutendste Planet ist, dał3 aber im Laufe der Zeit, eben weil man ihn als einen unbestimmten Planeten betrachtet, auf ihn die Eigenschaften der anderen Planeten aufgehäuft wurden; deswegen ist er z. B. weiblich wie Venus, männlich wie Jupiter etc.

2) Wie nah die griechischen Vorstellungen dem Inhalte der Leber verwandt sind, zeigt auch hier die Sage. Wie Hermes aus dem Berge Kyllenios geboren wird, so ist auf der Leber die Zunge Gottes, durch die verbindende Linie, als aus cilensl auftauchend dargestellt; bei beiden aus dem Gehöhlten, Verhüllten. 
(itus) nach Macrob. Sat. I 15 etruskisch. " "Vollmond“" und weiter Macrob. Sat. I 15, 14 ,sunt qui aestiment Idus ab ove iduli dictas, quam hoc nomine vocant Tusci et omnibus Idibus Iovi immolatur." Die ganze Stelle bei Macrobius Sat. I, 15, 14-17 läßt gar keinen Zweifel, daß hier „Vollmond" gemeint ist, ebenso wie "Schaf".

So steht also etruskisch „itus" für „Mond" und „Schaf", anstatt für "Planet" und „Schaf" im Babylonischen. Diese Übereinstimmung von "Mond" und "Schaf" zeigt wieder deutlich den Zusammenhang zwischen den babylonischen und etruskischen Auffassungen; andererseits aber dadurch, daßi die Babylonier den auf mehrere Planeten zersplitterten Begriff „Schaf" haben, während die Etrusker den einen einzigen Mond als "Schaf" bezeichnen, daf der Inhalt der Leber sehr wahrscheinlich der ältere ist, wie ich schon oben sagte ${ }^{\mathbf{1}}$ ).

1) Ich kann nicht umhin, hier auf etwas hinzuweisen, was sich mir erst nach Abschluf der vorliegenden Arbeit ergeben hat und was auf die Verbindung der Völker ein eigenes Licht wirft. Es scheint nämlich, daß die interessante Feststellung Jastrows von der Übereinstimmung des Wortes "Schaf" und "Planet" mit Zunge oder Sprecher sich nicht nur für das Babylonische nachweisen läßst. Vom Etruskischen itus $=$ Schaf $=$ Mond sagte ich schon. Nun aber scheint sich mir aus einer bis jetzt noch nicht völlig abgeschlossenen Untersuchung zu ergeben, daß auch in den germanischen Sprachen und Dialekten regelmäßig Schaf $=$ Mond oder Schaf $=$ Gestirne durch dasselbe Wort ausgedrückt wird. Wo aber Planeten "Schafe“, also „Sprecher" der Götter genannt werden, dort müssen "Schafe" an sich als Sprecher der Götter benutzt worden, also Leberschau betrieben worden sein. Und da auch im Chinesischen das „kwa Tui" für „Schaf“, „Vermittler zwischen den Menschen und Göttern“, und „Mund" dasselbe ist, so zieht sich hier ein Faden von China bis nach dem äußersten Norden und Westen Europas von solcher Stärke, daß an Zufall wohl kaum zu denken ist. Ich werde auch dieses in dem Nachtrag zur vorliegenden Arbeit näher besprechen. Jedenfalls erweist sich Jastrows Abhandlung "Name and Sign for Planet in Babylonian", von ungeheuerer Wichtigkeit. Ich kann nicht umhin, hier auf eine chinesische Úberlieferung aufmerksam zu machen, die bei de Groot, Religious-System of China, Book II, Vol. V, S. 495 zu finden ist: „It is related in the Kwoh yii that Ki Hwan-tszĕ, a grandee of the state of Lu caused a well to be dug, when they fetched up something like an earthen pot with a goat in it." (Nach persönl. Mitt. von de Groot ist im Chinesischen das Wort für Schaf und Ziege dasselbe und gerade diese Stelle bedeutet nach de Groot „Schaf“). „He had Confucius interrogated about it in these words: I dug a well and got a $\mathrm{dog}$; tell me what it is. On which the Sage answered: „According to what I have learned it must be a goat; for I have heard that apparitions between trees and rocks arc called khwei and wang-liang, while those in the water arc lung or dragons and wang-siang, and those in the ground are called fen-yang." (Schaf). Hierzu findet sich dann in demselben Buche S. 535 eine andere Lesart: Ngai, the ruler of Lu ordered a well to be dug. In three months they had not yet reached the water springs, but they found a living goat. The ruler ordered his invokers to operate on it with drums and dances in order to send it up (as a sacrifice?) to heaven, but it could not be sent up. Confucius saw the animal and said: ,the vital spirit of water is jade stone, and that of earth is a goat, so this goat must have an earthen liver." The ruler ordered the animal to be killed, and they found its liver to be of earth". 
Auf der Leber scheint dem $\vartheta$ etlvm $\vartheta$ (der Zunge, dem Sprecher, dem Werkzeug uud der Waffe Gottes) große Wichtigkeit beigelegt zu sein, wie sich aus der Stellung ausweist. Zur Hälfte ragt er in die breite Mündung des Todesstromes hinein; zur Hälfte in die ungefähr ein Viertel des ganzen inneren Raumes der Leber einnehmende Region, aus welcher sich der Götterberg erhebt und in welche die Worte: $\operatorname{tins} \boldsymbol{\vartheta}$ ne $\boldsymbol{\vartheta}$ (Deecke und Thulin 15'), ca 9 a $\left(6^{\prime}\right)$, tlusc (14') mar (von Deecke zu tluse gezogen, ebenso von Körte) und mari (18) eingraviert sind.

Von diesen Worten habe ich tins 9 ne 9 und ca 9 a schon S. 12 und 13 besprochen und beide als Erklärungen für die beiden Hauptverkörperungen des Sonnengottes angenommen, übereinstimmend mit Thulin, Götter des Mart. Cap. S. 50.

Das Wort tluse ist dadurch, daß man es nur richtig lesen kann, sobald man die Leber so hält, daßs das Loch in dem Götterberge dem Beschauenden zugewendet ist, als Unterschrift zu diesem charakterisiert. Erklärt ist es bisher noch nicht; doch nehmen sowohl Deecke als Thulin an, daß es identisch mit Consus sei, weil die Reg. X des Martian (unter 5 andern Götterbezeichnungen) auch den Consus enthält (s. Thulin, Götter des Mart. Cap. S. 55).

Die Zeichnung sagt folgendes aus: Dasselbe tlusc, welches in Reg. $10^{\prime}$ der Mitte der südlichen Leberhälfte als „sterblich“ gezeichnet ist, weil es dem Brunnen des Lebens (dem Kreise inmitten der südlichen Leberhälfte s. S. 31) entsteigt und welches in der Form tlusev in Reg. X des Randes als „unsterblich" gezeichnet ist, weil es daselbst ein Stück der Schlange (also der Ewigkeit) bildet dieses selbe tlusc vermag sich auf dem Götterberge zu zeigen, indem es aus seiner unterirdisch gedachten (weil südlich, also jenseits des Todesstromes gelegenen) Wohnung durch einen ihm eigens vorbehaltenen Ausgang auf dem Hange des Götterberges zu erscheinen vermag. tlusev stellt also ein vielfach Vorhandenes vor, das gleichsam unterirdische Botendienste zum Götterberge verrichtet. Soweit ohne Worterklärung.

Mit Worterklärung, ohne die man hier nicht auskommen kann, muß man tlusc als „zufällig“ deuten, wie ich glaube, während das Wort tluscv aus Reg. X wie mir scheint, „Zufälle" heißst.

Was wir uns unter diesem tluse zu denken haben, scheinen die etruskischen Sagen zu erklären: Deecke, Etrusk. Forsch. IV S. 42,

Diese Erzählung ist späte Überlieferung. Aber sie wird wichtig durch die völlige Übereinstimmung der $8 \mathrm{Kwa}$ mit dem Inhalte der Bronzeleber, sowie durch die Einteilung des geomantischen Kompasses der Chinesen in 16 Regionen, deren Inhalt ebenfalls mit dem der 16 Randregionen der Bronzeleber übereinstimmt. Ich muß leider die Zusammenstellung ebenfalls in den Nachtrag zu dieser Arbeit verweisen, da ich das Material dazu erst erhielt, nachdem diese Arbeit fertig vorlag. 
berichtet von dem, dem Tarchetius erschienenen Phallus, der plötzlich aus dem Herde auftauchte, von einer ähnlichen Sage von Servius Tullius und von einer Abbildung aus einem etruskischen Grabe; auch der Zwerg Tages taucht plötzlich aus dem Boden, um ebenso wieder $\mathrm{zu}$ verschwinden.

So scheint auf der Leber das Loch an der Seite des Götterberges (dessen Abbilder die aus Steinen erbauten Altäre, die heiligen Herde darstellen), zufälligen, rasch verschwindenden, vielfach trügerisch gedachten Erscheinungen göttlicher Herkunft als Aus- und Eingang zu dienen. Ja, wir werden vielleicht, falls meine Worterklärungen richtig sind, annehmen dürfen, daf tluscv in Reg. X "Zwerge" (Kobolde, Wichtelmännchen) darstellen, da sich eine Anzahl von Worten aus der Wurzel tlus entwickelte, welche darauf hinweisen.

Es bleiben von den in diese große Region eingeschriebenen Worten nur mar und mari, als auf diesen Raum bezüglich und zur Erklärung dienend übrig, weil tlusc sich nur auf das Loch im Götterberge bezieht und ne $\vartheta$ und ca $\vartheta$ a nur insofern auf diese Region Bezug haben, als sie dieselbe durch ihre daselbst stattfindende Vereinigung, jenseits des Götterberges, wo keine Linie sie trennt, als die "feuchte" charakterisieren, weil Hitze (ca9a) und naß (ne 9 ) zusammen warme Feuchtigkeit geben. Der Begriff einer großen warm-feuchten Ebene am Fuße eines Berges muß notwendigerweise eine Wiese, ein Anger sein; und da finden wir in der griechischen Sage die Asphodelos-Wiese, in der germanischen den Volkvangr, als Aufenthaltsorte der Seelen vor ihrer endgültigen Verteilung in die verschiedenen Regionen der Unterwelt.

Daß auch auf der Leber dieser Raum der gleichen Bestimmung zugeschrieben ist, zeigt das Wort mari (Thulin Reg. 18), welches nach meinem System der Worterklärung „die mit dem Mal Gezeichneten" „die Ausgezeichneten" zu bedeuten seheint, sowie mar "Mal" oder "Marke".

Aber nehmen wir das, was die Zeichnung ohne Worterklärung ausdrückt.

Daß mar und mari nicht das im etruskischen für den Gott Mars gebrauchte maris oder mariśs ist, muß auch der Unbefangenste einsehen; dagegen ist ebenso klar, daß mar und mari etwas dem Gotte maris oder mariś Ähnliches bedeuten.

Mari ist bei Thulin mit Reg. 18, das daran grenzende herc mit 19 beziffert. Das ist insofern nicht richtig, als im allgemeinen jede Zählung auf der Leber entweder der Schriftrichtung, also von rechts nach links, oder dem Laufe der Gestirne, also von Osten nach Westen folgen muß, außer dort, wo durch die Buchstabenrichtung eine Ausnahme ausdrücklich bestimmt ist, um damit eine feindliche Bewegung anzudeuten. (S. unten S. 39.) Es muß demnach herc 
mit 18, mari mit 19 beziffert werden, was zugleich darstellt, daß aus einem älteren (weil weiter östlichen) herc ein jüngeres mari hervorgeht oder sich an herc anschlieist. Das stimmt mit den Spiegeln (s. Thulin, Götter des Mart. Cap. S. 26) die man dahin deutet, daß nach etruskischer Auffassung Mars der Sohn des Herkules war, was sehr wohl mit der Auffassung aus der Zeit der Spiegeldarstellungen stimmen kann. Für den Leberinhalt müssen wir aber eine bedeutend frühere unpersönliche Auffassung annehmen, so wie es die Worte vorschreiben. Die Zeichnung gibt an: mars-ähnlich kommt später als herkules-ähnlich; beides steht auf der im Sonnenlichte liegenden Erdoberfläche, aber am Ufer des Todesstromes, überragt von der Gallenblase (in welcher ich mit Deecke zugleich das symbolische Bild einer Keule sehe. S. S. 33, 34), was also nur heißen kann: herc und mari sind untertänig oder abhängig von der Keule, so wie man dieselbe entweder als Attribut des Herkules, oder besser und älter als das des Sonnengottes (wie es z. B. auf den etruskischen Münzen festgehalten ist), auffaßt. Da außerdem mari als von herc kommend und an dem schmalen Durchgang, der zwischen dem großen Anger und dem Todesstrom von dem Processus papillaris, also der Zunge Gottes bewacht wird, endend, dargestellt sind, so scheint das in der Sprache der Leber zu heißen: mari warten auf dem großen Anger, bis durch die Waffe, das Werkzeug oder die Zunge Gottes das Malzeichen zum Übergang über den Blutstrom gegeben ist.

An dieser Stelle zeigt die Leber die Zunge (Waffe, Werkzeug, Willen) Gottes, der die Zeit erhellt $=$ Y $\operatorname{etlvm} \mathcal{\vartheta}=$ Mond (nämlich „Y lvm‡" als Zeitwort, „itus“ im Sinne des am Vollmondstage „sprechenden" Gottes) als Geleiter der Seelen über den Todesstrom und zeigt zugleich, daß von hier aus sich die Figur des etruskischen Charun, des Hermes-Psychopompos gebildet hat, indem man für diese Funktion des göttlichen Werkzeuges eine eigene Personifikation erfand. Es scheint, als ob die Leber noch einen andern Punkt aufwiese, der diese Annahme bestätigen dürfte. Das rechte, der lichten Erde und dem Anger der Seelen zunächst liegende Ufer des Todesstromes (welches dem vom Nabel zur Leber zuströmenden Ductus venosus entspricht) scheint sich jenseits der Brücke, unter welcher der Todesstrom entspringt, in die südliche unterirdische Leberhälfte in einer Linie fortzusetzen, welche in dem Kreise endigt, welcher seinerseits die Mitte der Unterweltsregionen bildet.

Als Zeichnung scheint dieser, mit einer Linie im Innern versehene Kreis ein Auge darzustellen, während Sagen und Märchen uns lehren, in diesem, uns aus der Tiefe des Erdinnern anschauenden Auge einen Brunnen zu sehen. Wir wissen von Odin, der seiner eines Augen in den Brunnen der Urdh (d. i. des Werdens) werfen muf, um allweise $\mathrm{zu}$ werden; wir wissen von dem Brunnen, aus welchem die Seelen für die Neugeborenen geholt werden, und wir wissen 
von den brunnenförmigen Gräbern, in denen die Etrusker ihre Blitze bestatteten, indem sie die Waffe Gottes wieder in den Schoß der Erde versenkten, aus dem sie gekommen war. Gerade diese letztere Utberlieferung, eben weil sie etruskisch ist, zeigt die engste Beziehung zum Leberinhalte.

Es scheint nämlich, daß genau so, wie die ältesten Darstellungen des Hermes (Säulen mit einem Haupte und dem Abbilde der Kraft) symbolisch für die geistige und körperliche Kraft Gottes stehen, so daß sowohl Sprache und Gesetz, als auch die körperliche Erschaffung des neten menschlichen Geschlechtes (im Gegensatze zum alten, vor der Flut) von ihm ausgehen - daß genau so der Brunnen, aus welchem das Werkzeug Gottes sich wiederspiegelt, als geistiger und körperlicher Schoß der Erde angesehen werden muß, aus welchem sowohl die Seelen als auch die Hüllen der Geborenen vermittelst des göttlichen Werkzeuges zum Lichte emporsteigen. Auch auf den babylonischen Lebern findet sich dieser Begriff „Schoß“, aber ebenso wie bei dem "Schaf" (s. S. 25) wie es scheint in der Mehrzahl. Aus dieser engen Beziehung zwischen \etlvmy (Processus papillaris) und dem Brunnen scheint die symbolische Bedeutung des Merkur, sowie das Variieren der Bezeichnungen für den Mond und den Planeten Mercur zwischen männlich und weiblich hervorgegangen zu sein. Außerdem scheint dieser Weg des Seelengeleiters Mond auch zugleich als der Weg der Sonne, den sie nachts vom Westen zu ihrem Aufgang im Osten durchläuft, gedacht zu sein.

Durch die Anordnung der Worte, welche als Erklärungen in die Kreisausschnitte graviert sind, die den Brunnen umgeben, scheint ausgedrückt, daß drei derselben dem Brunnen entstiegen, also aus ihm geboren sind, weil sie mit ihrem Anfang dem Brunnen zunächst stehen; drei andere sind dargestellt, daß sie von ihrem außen liegenden Anfang zum Brunnen hingehen, also aus ihm schöpfen, aber nicht von ihm geboren sind.

Die Schöpfenden sind: cilen, welche durch ihre Stellung außerdem als Schwester von fufluns, Tochter von cav und here gezeichnet ist s. S. 20 ; satres und lvsl vel.

Die dem Brunnen Entstiegenen, also aus ihm Geborenen sind: tlusc, le $\boldsymbol{\vartheta} \mathrm{ms}$ und selva. Da aber zu einer Deutung dieser Worte ohne Etymologie gar keine Anhaltspunkte gegeben sind, so lasse ich dieselbe hier fort (s. die Deutungsversuche bei Thulin, in seinen Göttern des Mart. Cap.)

Was ich ausdrücken wollte, ist dieses: daß der Brunnen aller Weisheit und allen Werdens, der zugleich der Schoß der Erde ist, durch einen Weg, welcher aus der Unterwelt über die Brücke und von dort am Ufer des Todesstromes weiter führt, mit der Zunge oder der Waffe Gottes, die zugleich die symbol. Leber ist, verbunden ist. 
In der Sprache der Leber: daß der „Sprecher Gottes“, der auf der Leber der Mond ist, der später zu Charun, Hermes, Merkur geworden ist, ebensowohl die Seelen der Toten zur Unterwelt geleitet, als die Seelen der Neugeborenen aus dem Brunnen über die Brücke in die Sonnenseite bringt.

Von den in die große Region, welche ich mit der Asphodeloswiese oder dem Volkvangr verglichen habe, eingeschriebenen Worten bleibt jetzt nur noch das bei Thulin in Reg. 14 stehende mar zu besprechen.

Thulin nimmt an, daß die wunderliche Anordnung der Buchstaben von einem Versehen des Schreibers herrühre, (Götter des Mart. Cap. S. 21); „nämlich ein vertikal gegen die Randregion geschriebenes ar und darunter ein horizontal geschriebens m."

Da aber in alter wie in neuer Zeit alles, was graviert werden soll, erst vorgezeichnet werden muß, ehe der Grabstichel in Funktion tritt und da man annehmen darf, daß3 bei Anfertigung eines so wichtigen Gerätes wie die Leber große Sorgfalt aufgewendet wurde, so glaube ich, daß diese Anordnung der Buchstaben absichtlich geschehen ist; und zwar deshalb, um damit den wirbelnden Flug der göttlichen Waffe anzudeuten: ihr glänzendes Dahinschießen und ihre Rückkehr zum Ausgangspunkte ${ }^{1}$ ).

Unwillkürlich fallen uns dabei die schwarzblau glänzenden Vögel ein, z. B. der Specht und die Taube, die als Vogel des Mars oder als Beilvogel der Ischtar bekannt sind und welche diese Beziehung zu den Göttern wohl nur ihrem, der geschleuderten Waffe gleichenden zickzackig dahinschießenden Fluge verdanken. Sie symbolisieren gleichsam dieses wirbelnde mar von der Leber.

Buchstäblich scheint mar „Mal" zu heißen, „Marke“ oder „Merkzeichen". Der römische Mars müßte danach in seiner ältesten Vorstellung den Begriff des vom Gotte geschlagenen Males, also der Verwundung enthalten; mit anderen Worten: der Gott Mars müßte die personifizierte, tötliche, vom Gotte geschlagene Wunde sein; das göttliche „Mal par excellence", woraus sich dann der Begriff des Kriegsgottes erst entwickelt hätte. (S. Preller, Mythol. S. 252, 253.)

Wir kommen nun zu der Gallenblase.

Als Zeichnung an sich betrachtet scheint sie eine Keule, einen Keil, einen Pfahl, oder einen Nagel zu bedeuten und ist, weil die breite Basis teils auf der. Schlange, teils auf der, als im Lichte liegenden Erdoberfläche gedachten, nordöstlichen Seite der Leber aufliegt, als teils der Schlange, teils der Erde entsprossen gezeichnet. Es ist dieses weder schwierig zu sehen, noch schwierig zu deuten. Ebenso

1) Auf die in die Hand des Schleudernden zurückkehrende Waffe weisen die Bumerangs der Ägypter, die Sagen von Thors Hammer und die etruskische Blitzbestattung, welche den Blitz, die Waffe Gottes, zur Erde, aus der er gekommen ist, zurückkehren läßt. 
deutlich ist dargestellt, daß die über mari und fuflus hinwegragende Spitze diese beiden (mari und fuflus, die ich mit "Malversehenen“ und "Wölfischen" übersetze) als von eben dieser Spitze der Gallenblase beherrscht darstellt. Wie aber könnte man erklären, als was die Gallenblase sonst noch aufzufassen ist, wenn nicht durch die Erklärung der Worte in denjenigen Regionen, an denen die Gallenblase „festgewachsen“ ist, was in der Sprache der Leber soviel heißt, als „in unlöslichen Beziehungen steht."

Auf der nördlichen, also der Wohnung Gottes näher gedachten Seite ist die Gallenblase festgewachsen an - oder in unlösliche Beziehung gesetzt zu - lasl $\left(4^{\prime}\right)$ le 9 n $\left(2^{\prime}\right)$ nc $\left(3^{\prime}\right)$, die alle drei dadurch, daß sie nördlich, also im Bereiche des Götterberges liegen, zwar als zu Gott in Beziehung stehend, aber dadurch, daß sie nicht auf die Schlange (also ewig), sondern auf die Oberfläche der Erde gezeichnet sind, als sterblich dargestellt sind.

Auf der südlichen Seite ist die Gallenblase festgewachsen von da, wo cav und here sich verbinden, bis dort, wo herc zu Ende ist und an mari anstößt. Das scheint in der Sprache der Leber: weil der größte Teil der "Keule" oder "des Holzes" mit „erdig, irdisch" (oder, wenn man lieber will, mit ,herkulesähnlich“) verbunden ist, so ist sie vorwiegend erdig gedacht und ebenso als unauflöslich verbunden mit der Vorstellung der Vereinigung von ca' und herc. Weil aber die Keule nicht, analog dem gesetzmäßig von rechts nach links laufenden Wege der Schrift folgend, aus der Vereinigung von ca $\boldsymbol{\vartheta}$ und herc entspringt, wie es die Schlange, fufluns und cilen tun, die dadurch als gesetzmäßig aus dieser Vereinigung entsprungen gezeichnet sind, sondern: weil die Keule dem Verlauf der Schrift (also dem Gesetze des Verlaufes) entgegensteht, ist sie als ungesetzmäßig dieser Vereinigung von ca $\vartheta$ und herc entsprungen dargestellt. Das kann aber keinen Tadel in sich schließen, weil die Keule an sich als „glücklich“, weil im Osten wohnend, gedacht ist, sondern kann wohl nur einen Rang-Unterschied gegenüber den gesetzmäßig Entsprungenen bezeichnen.

Das Schwergewicht, die Basis der Keule, also symbolisch: ihre größte Bedeutung liegt über den Regionen le 9 n (4 und e $\boldsymbol{9} 5$ ) des Randes, die beide dadurch, daß sie auf die Schlange geschrieben sind, als unsterbliche Stücke Gottes bezeichnet sind, für deren Deutung aber ohne Worterklärung gar kein Anhalt gegeben ist.

Deecke und Thulin sehen in e $\boldsymbol{9}$ die Abkürzung von e 9 ausva (wie in tin tinia, in ne 9 ne $\mathscr{Y}$ uns, in here hercle etc.) und identifizieren sie mit Ceres (s. Thulin, Götter des Mart. Cap. S. 48). Leףn, das bei Deecke und Thulin nur eine Form von le $9 \mathrm{~ns}$, le $9 \mathrm{~ms}$, le 9 am und leta ist, wird als Kriegsgott gedeutet (s. Thulin, Götter d. Mart. Cap. S. 43, 44); aber die Begründung dafür (nämlich einerseits das Vorkommen des Wortes auf der Bronze an und für sich und 
andererseits, weil die Kriegsgötter bei den Etruskern eine große Rolle gespielt haben müssen, da sie der Tradition nach einstmals fast das ganze Italien beherrscht haben), ist nicht zwingend.

Ich sehe in leyn "Geleiter" oder "Glieder" und zwar hier, da sie auf die Schlange geschrieben sind, unsterbliche und in ey "irdisches" oder „Herd-Feuer" ${ }^{1}$ ).

Es scheint aus der Beziehung der Gallenblase zu den sie umgebenden Regionen (falls meine Deutung der Worte richtig ist) hervorzugehen, daß die Keule, oder das Holz mit Feuer und mit Wasser als verbunden gedacht ist; mit Feuer durch die sie umgebenden Regionen, mit Wasser dadurch, daß ihre Basis auf der Schlange liegt und durch das unter den 5 Aufschriften auf ihr selbst geschriebene „n“, welches sich, wie ich glaube, durch ne $\boldsymbol{Y}=$ naß deuten läßt.

Die Keule scheint aber nicht mit dem himmlischen Wasser und dem himmlischen Feuer verbunden, wie der Götterberg es durch das Niederlassen Gottes aus dem Himmel als ney und caya ist, sondern mit dem Wasser und Feuer, wie es sich auf der im Lichte liegenden Erdoberfläche findet, also mit dem Wasser der Quellen und Flüsse und dem aus Holz und Stein gewonnenen Feuer; beides beherrscht oder enthält die Gallenblase in guter und schlimmer Bedeutung, genau wie der Götterberg (der „Finger“ Gottes, oder das „Horn der Hand" s. Ja strow, Relig. Babyl. u. Assyr. II S. 231) das Nasse und Heiße im guten und im schlimmen beherrscht.

Es scheint überhaupt, als ob die Gallenblase in der Form und dem Sinne nach die irdisch gedachte, symbolisch in dem Baume oder Holze des Lebens ausgedrückte Schöpferkraft Gottes, wie sie sich in allem, was lebend aus der Erde hervorgeht, dokumentiert, darstellen soll; während der Götterberg, das Haupt und Horn, die himmlisch gedachte, (symbolisch im Steinhaufen, Altar, Herd, Steinkeil ausgedrückte) Schöpferkraft Gottes, wie sie sich in allem dokumentiert, was zwischen Himmel und Erde hervorgeht, also Blitz, Donner, Hagel, Regen, Wind, Personifikationen Gottes, Halbgötter, Dämonen, Erscheinungen, Träume, Orakel, Leben an sich, Tod an sich, Sieg, Verstand, Willen usw. darstellen soll ${ }^{2}$ ).

1) Siehe auch S. 38 .

2) Wahrscheinlich erklärt die Leber hier auch das Einschlagen der Nägel in die Tempelpfosten, sowie den Kultus des Nagels an sich, wie er z. B. in Tölz in Bayern als Verehrung des St. Leonhards-Nagels bis heute besteht, oder in Franken, wo die Bauern bis heute Nägel in die Türpfosten der Ställe schlagen, um Hexen und anderes Unheil abzuwehren; oder auch, um auf einen der ältesten Beweise für die Verehrung des Nagels zurückzugehen, sei hier der Götterfiguren aus Bronze gedacht, die in einen langen Nagel auslaufen und welche in den ältesten Schichten von Tello im Fundamente von Bauten (in Babylonien) jetzt gefunden sind (s. Eduard M e y e r, Geschichte des Altertums, I. Bd., 2. T., S. 416).

Da die Keule auf der Leber aus Holz gedacht scheint, so müften deren Abbilder, die kleinen Keulen oder Nägel ursprünglich auch als hölzern gedacht 
Nach dem Befund von der Leber, woselbst das Attribut der göttlichen Schöpferkraft gleich starke Beziehungen zu Feuer und Wasser hat, müssen die Nägel hauptsächlich zur Abwehr von Feuerund Wassergefahr in die Türpfosten geschlagen worden sein und zwar in dem Sinne, daß das aus dem Wasser und irdischen Feuer stammende Symbol der Schöpferkraft Gottes das von ihm beherrschte irdische Feuer und Wasser zurückhalten möge.

Es ist uns ein etruskischer Brauch überliefert, welcher Bezug hierauf haben kann: „arse verse averte ignem significat. Tuscorum enim lingua arse averte, verse ignem constat appellari. Unde Afranius ait „Inscribat aliquis in ostio arse verse" (s. Thulin, Etrusk. Disziplin I S. 126).

Ich komme nun zu den Inschriften auf der Gallenblase, die Thulin, Etrusk. Disziplin II S. 20 u. 21 mit den Inschriften der babyl. Leber zusammenstellt und zwar so, daß er bei der Spitze beginnt. Das erscheint insofern nicht richtig, als das göttliche Attribut, für welches die Gallenblase zu gelten scheint, als zur Schlange zugehörig gezeichnet ist, weil die breite Seite dort festgewachsen dargestellt ist; ganz abgesehen davon, daß alle Lesungen, die nicht von rechts nach links nach der Richtung der Schrift laufen, von Osten nach Westen gelesen werden müssen. Deshalb muß eine sinngemäße Erklärung an der Basis beginnen ${ }^{1}$ ).

Ich will keine Lesung der fünf Abteilungen der Gallenblase hierher setzen, weil dieselbe, da hier der Raum fehlt, um Beweise dafür zu geben, überflüssig ist.

Die Basis der Gallenblase enthält ein verstümmeltes Wort, von welchem die bisherigen Lesungen die Buchstaben tv ? $\&$ festgestellt haben. Ich möchte hier eine Vermutung wagen. So gut in tin die Wurzel für tina, in ne $\vartheta$ die Wurzel für ne $\mathcal{Y}$ uns vorzuliegen scheint, so gut scheint in diesem tv ? $\boldsymbol{\vartheta}$ die Wurzel der unzweifelhaft festgestellten Götternamen turms und turan vorzuliegen, sobald die Lücke mit einem $r$ ergänzt wird. So gut tinia buchstäblich „Abzweiger" oder "Teiler" heißt, neduns "Nasser"; so gut scheint turms, der etruskische Name für Merkur, „Donnermann" zu heißen und turan, der etruskische Name für Venus, „Donnerin“. Ich werde dieses in meiner ausführlicheren Arbeit besser ausführen. Sollte ich recht haben, so würde das tv (r) $\vartheta$ der Gallenblasenbasis bedeuten „er donnert",

werden, was durch das Übereinstimmen von lat. clâva $=$ Keule, clâvus $=$ Nagel, clavis $=$ Schlüssel; griech. Erle, Schloß und Riegel $(x \lambda \dot{\eta} \vartheta \varrho \eta$ und $x \lambda \tilde{\eta} \vartheta$ @os $=$ Erle, $x \lambda \tilde{\eta} \vartheta \rho o \nu$ und $x \lambda \dot{\eta} \ddot{\imath} \vartheta \varrho o v$ ion. was $x \lambda \varepsilon \tilde{\imath} \vartheta \rho o \nu=$ Schloß oder Riegel, claustrum) unter anderem bestätigt wird. (S. auch Schrader, Reallexik. der indogerm. Altertumskunde sub Nagel).

1) Auch in der babylonischen Leberschau wird bei Zerteilung der Gallenblase, sowie bei den anderen Teilen der Leber immer von der Basis begonnen. (S. Jastrow, Relig. Babyl. u. Assyr. II, S. 254, Anm. 3. 
wie z. B. diese Form in th endend in "tins $\boldsymbol{\vartheta}$ ne $\boldsymbol{\vartheta}^{\text {" }}$ auf der Leber schon einmal vorgekommen ist, wo die vollständige Übersetzung lauten müßte „er schwillt naß̈.

Auf marisl, lay und leta will ich nicht weiter eingehen, um nicht unnötig das etymologische Gebiet $\mathrm{zu}$ streifen; möchte aber nochmals hervorheben, daß die Ansicht der Etruskologen, die in le $\mathcal{Y}$, leta, le $\boldsymbol{\vartheta}$, le 9 ns und le 9 am ein und dasselbe Wort sehen und es als einen und denselben Kriegsgott deuten, kaum das Richtige trifft.

Die vierte Region enthält ein in der Richtung von Osten nach Westen geschriebenes $\mathrm{n}$, während die übrigen Worte der Gallenblase von Norden nach Süden laufen. Dieses $n$ ist, weil es, anstatt mit den unteren Inschriften der Gallenblase gleichzulaufen, quasi aus denselben herausströmt, auch in solchem Sinne aufzufassen. Auch ist es, wie ich glaube, nicht zu neYuns zu ergänzen, wie Thulin es tut, sondern zu ne $\boldsymbol{\vartheta}$; obgleich der Sinn der Bedeutung dadurch nicht wesentlich geändert wird, ne $\boldsymbol{y}$ uns ist nur insofern auszuschließen, als er tatsächlich auf der Leber nicht existiert.

Die Spitze der Gallenblase ist nur mit dem Buchstaben $\boldsymbol{y}$ bezeichnet, dessen Deutung mit dem übereinstimmen muß, was auch in der Zeichnung und Stellung der Spitze ausgedrückt ist. Und da die Spitze als: „die im Lichte liegende Erdoberfläche beherrschend" dargestellt ist, während alles lebend aus der Erde Hervorgehende als aus der Keule stammend dargestellt ist, so muß dieses $\vartheta$ die Beherrschung alles Lebenden auf Erden in einem Worte zusammenfassen, welches die Gewalt über Leben und Tod zugleich ausdrückt, also Erzeugung und Vernichtung durch Gott.

Ich kann nicht umhin, bei Besprechung der bildlich dargestellten vier Zeichen von der Leber (Götterberg, Zunge, Keule und Brunnen) noch eine Übereinstimmung mit asiatischen Vorstellungen zu erwähnen, aus welcher, neben der Úbereinstimmung auch abermals hervorzugehen scheint, daß der Inhalt der Leber die älteren Begriffe enthält.

Eduard Meyer, Geschichte des Altertums I. Band, 2, S. 466, sagt von den Amoritern: "Als Hauptgott verehren sie einen Gott Hadad, der im Gewitter dahinfährt und den Blitz, oder auch einen gewaltigen Hammer schwingt. Die Akkadier haben ihn übernommen und bezeichnen ihn häufig mit dem Beinamen Ramânu, oder „Brüller“ (Donnernde). Von ihnen kam er zu den Sumeriern, Lulubäern und Assyrern. Neben ihm Amurru in kriegerischem Gewand, in der Rechten einen gebogenen Stab, vielleicht eine Art Wurfholz, Speer in der Linken; gelegentlich tritt er auf einen Hirsch, oder ein Steinbock steht hinter ihm. Später oft mit Hadad identifiziert und vielleicht ist es nur ein anderer Name des Gottes. Seine Gemahlin ist Ašera (Ašrat), d. i. das göttliche Wesen), das in dem am Altar des Gottes aufgerichteten heiligen Pfahl haust." 
Deutlich sehen wir hier ca9a von der Leber, die hitzige Verkörperung des obersten Gotherrn, wie er sich bei dem steinernen Götterberge als Schleuderer dessen, der die Zeit erhellt, $\boldsymbol{\vartheta}$ etvlm $\boldsymbol{\vartheta}$ (Zunge, Blitz, Hammer, Axt) dokumentiert, zu einem persönlich gedachten Gotte Hadad werden; wir sehen weiter $\vartheta$ etlvm $\vartheta$, die Zunge und Waffe Gottes zu einem persönlichen „Sprecher", dem Gotte Amurru werden; und sehen, wie das über Wasser und Feuer gebietende Holz, die Keule, sich in einen verehrten heiligen Pfahl, als Frau gedacht, verwandelt. Diese verwischten Begriffe, gegenüber den scharf umrissenen von der Leber zeigen deutlich, wieviel jünger sie sind. Doch ist ihr gemeinsamer Ursprung und ihre enge Verwandtschaft mit der Leber gar nicht zu verkennen.

Es bleiben jetzt noch die sechs von Norden bis Osten reichenden Randregionen der Leber und die in dem Raume zwischen dem nördlichen Leberrande und der Gallenblase liegenden Innenregionen zu besprechen. Von diesen muß für die sechs Randregionen die von Deecke angegebene und von Thulin beibehaltene Reihenfolge bestehen bleiben, während sich für die Innenregionen andere Beziehungen zu ergeben scheinen. Ich glaube nämlich, daß die vier ersten Randregionen durch die jedesmal daran anstoßende Innenregion genauer bestimmt werden, um Irrtümer möglichst auszuschließen, weil das System dadurch, daß sämtliche Erscheinungen auf der Leber schließlich immer wieder Erscheinungen des einen großen Gottes sind, ein verwickeltes ist. Es ist eben "Juppiter in allen Regionen", wie bei Martianus Capella, nur setzt er Juppiter für den einen großen Gott und Inhaber des Lichtes, usil.

Wenn aber die vier ersten Innenregionen sich ganz natürlich auf diejenigen Randregionen beziehen, die ihnen am nächsten liegen, so müssen sich auch die beiden übrigbleibenden Randregionen auf die beiden übrigbleibenden Innenregionen so bcziehen, daß3 die 5 ., eף, sich auf die zunächst übrig bleibende, das ist lasl (Deecke $4^{\prime}$ ) und die am weitesten von Reg. I abliegende Reg. VI, ca $\boldsymbol{\vartheta}$, sich auf die am weitesten vom Rande abliegende Innenregion, das ist fuflus (Deecke $5^{\prime}$ ) bezieht; was dann wieder mit deren Reihenfolge von Osten nach Westen stimmt.

Wir bekommen jedesmal (einmal im Rande und einmal inmitten der nördlichen Leberhälfte) die „Wölfischen“ unterhalb des „Heißen“

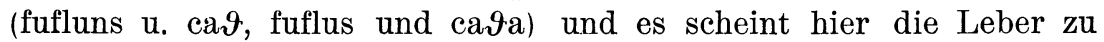
erklären, weshalb die Wölfe mit Apollo und der Sonne in den griechischen Sagen zusammengestellt werden und woher in den germanischen Sagen von den Wölfen die Rede ist, die hinter Sonne und Mond herlaufen. Auf der Leber ist ihre Stellung fest umrissen: hier sind sie die haarigen Söhne des obersten Gottherrn, in seiner zweiten Personifikation als der Hitzige mit der Erde gezeugt; während die Sagen zwar eine Beziehung der Wölfe zur Sonne festhalten, aber 
in verwischten Umrissen; ganz der Entfernung vom Inhalte der Leber gemäß.

ey in Reg. 5 ist „Herdfeuer" oder „irdisches Feuer", wie ich schon S. 34 sagte, unter der Herrschaft der Gallenblase, also aller der Erde entstammenden Fruchtbarkeit; und ist durch die Innenregion lasl („Reich der leeren unbeseelten Hülle?“) als das aus dem Stein geschlagene Feuer, im Gegensatz zum himmlischen Feuer genau festgestellt. Wegen der Beweise hierfür muß ich immer wieder auf meine ausführliche Arbeit hinweisen, da hier der Platz mangelt.

Es ist unmöglich, die Randregionen zu erklären, ohne eine Deutung vorzuschlagen, weil sie sich durch die Zeichnung nur als Teile und Stücke des einen Gottes (Teile der allumfassenden Schlange) auffassen lassen, während die Worte selber bestimmen, als welche Teile, oder besser gesagt "Teilungen“ Gottes sie zu gelten haben. Da aber für eine Deutung hier der Platz mangelt, so setze ich nur das allernotwendigste hierher.

Weil es scheint, als ob die Regionen der lichten Erdoberfläche sich in einem, ihnen ähnlichen, aber düstern Sinne auf der linken, südlichen Leberhälfte, jenseits des Todesstromes wiederholen (so daß Reg. I der Reg. VII, die die Erste nach dem Überschreiten des Todesstromes ist, entspricht), so mußte ani (I) von der Lichtseite fufluns (VII) von der Nachtseite gleichstehen ; (der „Uralte“ (?) dem „Wölfischen“ (?) uni in II = selva in VIII ; (die große Mutter (?) dem unsterblichen Selbst Gottes (?) tecrm in III = le 9 ns in IX ; (das Bedeckte (?) den Geleitenden oder Gliedern (?); le $\vartheta n$ in IV = tluscv in X; (die Geleiter oder Glieder (?) den „Zufälligen" (?) (zwergischen Erscheinungen). e $\vartheta$ in V, das ich durch Herdfeuer deute, entspricht der auf der Leber in Reg. XI durch Rost zerstörten Abteilung, welche demnach eine verderbliche oder trügerische Wirkung des Feuers dargestellt haben muß. Sie enthält nur die Buchstaben ce. Ca $\vartheta$ in VI entspricht cvlalp in XII; (heiß (?) der Krankheit (?)). Doch möge man dieses, weil die Beweise mangeln, überschlagen.

Ich komme nun zum letzten Punkte meiner Besprechung der Leber; das ist; ob die Leber für das Vorkommen einer 9-Zahl von Blitzgöttern Anhaltspunkte bietet.

Wegen der 9-Zahl der Blitzgötter s. Thulin, Etrusk. Disziplin I, S. 22: „Der Blitzgötter waren 9, Juppiter aber hatte 3 Manubien, es gab also im ganzen 11 Manubien." Und wegen der Übereinstimmung dieser Zahlen mit den chaldäischen, s. Thulin, Götter des Mart. Cap. S. 81: „Für die Zahl 11 der etrusk. Manubien oder Blitzarten kann ich nur eine Parallele heranziehen: Serv. Georg. I, 33 „Aegyptii duodecim esse adserunt signa, Chaldaei vero undecim " Siehe auch Roscher, die Sieben- und Neunzahl im Kultus und Mythus der Griechen.

Thulin meint (Götter des Mart. Cap. S. 81), daß die Etrusker die chaldäische Lehre aufgenommen hätten und aus der chaldäischen 
Sternwahrsagung eine Blitzwahrsagung gemacht hätten. Das scheint nicht so. Die Leber enthält keine einzige andere Beziehung zu Himmelskörpern, als die zum Monde; sie muß also zu einer Zeit festgesetzt sein, zu welcher der Appell an die Mehrzahl der „Sprecher“ Gottes unbekannt war und das verweist abermals die Entstehung des Inhaltes der Leber (wohlgemerkt, nicht der Bronze selber) in eine sehr weit zurückliegende Zeit.

Auch in der babylonischen Astrologie spielt der Mond eine überaus wichtige Rolle, so daß sich auch hierfür die Vermutung aufdrängt, daß wahrscheinlich die Deutung der Planetenvorzeichen und gewiß die der Fixsternvorzeichen erst spätere Zutaten sind.

Auf die Frage, ob die Leber Anhaltspunkte für die 9-Zahl der Blitzgötter enthält, erhalten wir eine, ganz der verwickelten etruskischen Disziplin entsprechende Antwort.

Wenn wir nämlich die Inschriften, welche den schlangenförmigen Leberrand ider zugleich das Meer und die Ewigkeit ist) anfüllen, genau betrachten, so muß uns sogleich eine merkwürdige Anordnung ins Auge fallen: die Worte sind derart eingeschrieben, daß sie eine Kolumne von Norden bei Reg. I ani angefangen, über Osten nach Süden, eine andere von XVI $\vartheta v f$ angefangen über Westen nach Süden bilden, deren Wortenden in den Regionen IX und $\mathrm{X}$ gleichsam mit den Füßen zusammenstoßen. Da kein einziges Wort auf der Leber ohne Absicht hingeschrieben ist, so muß man auf jeden Fall hierin eine Bedeutung suchen.

Stellen wir die Regionen zusammen, aus denen die über Osten gehende, linke Kolumne gebildet ist:

$$
\begin{aligned}
& \text { I ani } \\
& \text { yne } \\
& \text { II uni } \\
& \text { mae } \\
& \text { III tec } \\
& \text { vm } \\
& \text { IV leभn } \\
& \mathrm{V} \text { e } \boldsymbol{y} \\
& \text { VI } \operatorname{ca} \vartheta \\
& \text { VII fuflu } \\
& \text { ns } \\
& \text { VIII selva } \\
& \text { IX le } \vartheta \text { ns }
\end{aligned}
$$

so erhalten wir 9 Regionen, die durch die gleiche Buchstabenrichtung, in welcher sie geschrieben sind, als neun, durch eine Gemeinsamkeit verbundene Stücke Gottes, der Schlange oder des Meeres, oder der Ewigkeit dargestellt sind, die von Gott, gleich der Schlange, deren Haupt der Haarige ist, mit der Erde erzeugt sein müssen, weil sie selber Stücke der Schlange sind. 
Die Leber sondert durch die Schriftrichtung ganz augenscheinlich 9 Regionen aus, in denen wir ziemlich sicher die bei den Etruskern verbürgten 9 Blitzwerfer sehen dürfen. Nach Thulin, Etruskische Disziplin I, S. 22 wurde aber angenommen, daß diese 9 Blitzwerfer 11 Manubien hatten, weil Juppiter über 3 Manubien verfügte. S. Thulin, Etruskische Disziplin I, S. 24-30, nach welchem die erste Manubia $=$ fulmen praesagum von Juppiter allein, die zweite $=$ fulmen ostentatorium, auf Anraten der Consentes, Complices, Consiliarii, die dritte $=$ fulmen peremptorium im Einverständnis mit den dei superiores et involuti von Juppiter geschleudert wurde.

Das scheint, auf die Leber bezogen, folgendes: Da Juppiter in den aus dem Etruskischen genommenen römischen Kultusvorschriften immer für usil steht, so muß die erste manubia, der große fulmen praesagum „den Juppiter allein vom ganzen Himmel senden konnte“ (Thulin, Etruskische Disziplin I, S. 24) eben usil, dem obersten Gott-Herrn zugesprochen werden: und da auf der Leber usil (= Juppiter im römischen Sinne) ganz persönlich als er selbst in ca9a und in fufluns auftritt, so müssen diese beiden, da sie usil sind, im römischen Sinne auch wieder Juppiter sein und die beiden andern Manubien wird man ihnen zuschreiben müssen. Es scheint die Erinnerung an die Darstellungen von der Leber in der Beschreibung der Manubien ganz deutlich vorhanden zu sein: die zweite manubia, fulmen ostentatorium ist schlimm und gut zugleich, ganz im Sinne der göttlichen Waffe, wie caঐa von der Leber sie schleudert; und in den 12 Consentes, Complices, Consiliarii wird man wohl die Inhaber der 12 Regionen von ani bis zur Mündung des Todesstromes, die alle, gleich ca $\vartheta$, Teilungen der Schlange sind, von denen aber die in Region $\mathrm{X}$, XI, XII (tlusev, ce ..... (zerstört) und cvlalp, weil ihre Namen nicht mehr in der Richtung der ersten neun laufen, nicht blitzwerfend sind, sehen müssen. Die dritte manubia, gleich dem fulmen peremptorium, dem schrecklichsten verderbenbringenden verheerenden Blitz, der mit Feuer kommt und den Zustand des Staates oder den des Einzelnen umgestaltet (Thulin, Etruskische Disziplin I, S. 28) ist ganz deutlich auf den Wölfischen in der Verhüllung dargestellten Gott (usil-Juppiter) gemünzt, der nach der Leber der erste der Verhüllten ist, und auf den die Beziehung zu den dei superiores et involuti beim Schleudern der dritten Manubia geht. So gut aber usil und ca 9a vom Himmel zur Erde kommend gedacht sind und fufluns von jenseits der Grenze der Unterwelt aufsteigend, so gut müssen auch ihre Blitze oder Manubien die gleiche Richtung einschlagen, und deshalb müssen die beiden ersten Manubien vom Himmel, die dritte Manubia aus der Erde geschleudert gedacht sein.

Die den neun blitzwerfenden Regionen gegenüberstehende Seite des Leberrandes enthält die Regionen XVI bis X rückwärts, also 7 Regionen. 
Daß unter diesen 7 bösen (weil über den Westen sich verbreitenden) Regionen die septem residui des Martianus Capella verstanden sind (s. Thulin, Götter des Mart. Cap. S. 64) scheint einleuchtend. Aber es ist kein Bezug auf die Planeten auf der Leber $\mathrm{zu}$ ersehen, während Thulin denselben nach Kopp für die septem residui annimmt.

Um sich vorzustellen, was unter diesen 7 bösen Regionen verstanden sein soll, darf man vielleicht die Worte, die Eduard Meyer, Geschichte des Altertums, I, 2, S. 369 von babylonischen und assyrischen Götterwesen braucht, auch auf diese 7 Regionen der Leber beziehen:

„Oft treten die Dämonen in Gruppen auf; vor allem die Siebenzahl gilt allen Semiten als eine geheimnisvolle Zahl, die daher bei magischen Sitten und Kultbräuchen, speziell bei Eidschwüren vielfach verwendet wird. Die spätere babylonische Theologie hat dann Sonne, Mond und fünf Planeten zusammengefaßt." - Denn tatsächlich scheint die Leber die, durch die Richtung der Buchstaben zu einer Siebenzahl zusammengefaßten Regionen als dämonische zu bezeichnen, ebenso wie die Neunzahl der Regionen, die durch ihre Buchstabenrichtung auf der linken Seite zusammengefaßt sind, auf der Leber als "Starke" bezeichnet sind, wodurch beide - die 9 Starken $=$ den Anunnaki, die 7 dämonischen $=$ den 7 (ursprünglich 5) Igigi - den babylonischen Götterbegriffen gleichgestellt werden.

Weil die Leber die 9 östlichen und die 7 westlichen Regionen ohne jeden Bezug auf Astrologie zusammenfaßst, so zeigt sich auch hierin wieder der Inhalt der Leber (möge die Bronze selber so jung sein, als sie wolle) als aus sehr alter Zeit stammend.

Um noch einmal eine Übersicht über das zu geben, was die Bronzeleber von Piacenza zu sein scheint, so möchte ich glauben: sie sei ein Dokument der Vorstellung vom Makrokosmus, welche einer Zeit angehört, die noch als Begriffe dasjenige handhabt, aus dem sich später die definitiven Gestalten der Götter der griechischen, römischen und germanischen Mythologie entwickelten. In der Entwickelung der jüdischen Religion zu einem reinen Monotheismus können wir den Versuch erkennen, die uralten Begriffe, wie sie die Leber enthält, wieder herzustellen.

Ein in zahllosen übereinanderliegenden Schlingen verlaufender Weg zeigt an der Hand der griechischen Mythologie die Verbreitung dieses Leberinhaltes von Asien nach Griechenland; vielfache Rückkehr und erneutes Vordringen, wobei die Begriffe immer mehr verchswimmen. Ein nicht so oft begangener Weg zeigt den Inhalt der Leber in der germanischen Mythologie lapidar und in einer gewissen Herbheit vielfach deutlicher, als in der griechischen erhalten, wenn auch die Figuren der "Götter" ebenfalls eine ungeheure Zeit zwischen den Ausgangspunkt und den Endpunkt legen. Auf den Zusammen- 
hang des Leberinhaltes mit den chinesischen Vorstellungen, wie sie im Buche Yih für die $8 \mathrm{Kwa}$ festgelegt ist, muß ich im Nachtrag zu vorliegender Arbeit zurückkommen.

Die Genauigkeit der Darstellung und die raffinierte Kontrolle einer Region (durch ihre Lage und Verbindung) auf die andere, läßt einenteils die hohe Heiligkeit des Gerätes vermuten; andererseits darf man annehmen, daß es zugleich als Lehrgegenstand für die jungen Leberschauer zu dienen hatte.

Sollte sich im Laufe weiterer Entdeckungen meine Ansicht bestätigen, daß die Leber als Symbol für einen gewissen Kultus gegolten hat, so würde sich überall dort, wo Lebern gefunden werden, dieser Kultus und damit eine sehr interessante Spur für den $\mathrm{Zu}$ sammenhang der Völker nachweisen lassen.

So gut die Göttergestalten von verschiédenen Mythologien aus den Begriffen der Leber herausgewachsen $\mathrm{zu}$ sein scheinen, so gut hat man sehr wahrscheinlich die viel späteren astrologischen Vorstellungen den Begriffen der Leber angepaßst, welche selber von den Gestirnen nur den Mond nennt.

Übereinstimmend hiermit ist das aus den babylonischen Divinationen sich ergebende Resultat, daß die Leberschaukunde, als die „populäre“ Form der Weissagung älter ist, als die Astrologie, die eine .,wissenschaftliche" Form der Weissagung darstellt. S. Jastrow Hepatoscopy and Astrology in Babylonia and Assyria S. 652 seq.

Das, was ich hier gegeben habe, ist nur ein kurzer Überblick über das, was die Leber zu enthalten scheint und was ich bei einem Vergleich mit den Göttern des Martianus Capella zu erweitern hoffe.

Irrtümer mögen mir mituntergelaufen sein; man braucht sie nicht zu fürchten, denn die Zeit und vereinigte Arbeit von Vielen wird sie ausweisen; immerhin aber hoffe ich durch diesen Versuch einer Darstellung der Bronzeleber von Piacenza in ihrer symbolischen Bedeutung einen neuen Weg gefunden zu haben. 


\section{Register der auf der Leber vorkommenden Worte.}

I. Auf der gewölbten Oberseite: usils $5,7,9,10,12,18,19,40$. tivr $15,16,17,18$.

II. Auf der beschriebenen Unterseite:

ani 38,39 .

cav $13,18,19,21,23,27,33,37,38,39$. саэа $12,13,18,19,28,29,37,40$. ce $38,40$.

(cilen 10, 11, 31, 33.

cilensl 11, $14 \mathrm{f} ., 18,24$.

cvlalp 24, 38, 40 .

eף 33, 34, 37, 38, 39 .

fufluns $7,15,18,19,31,33,38,39,40$.

fuflus 37 .

herc $3,5,18,19,21,29,31,33$.

lasl $33,37,38$.

la 36.

leta 33,36 .

le 9 am 20, 33 .

le $\vartheta \mathrm{ms} 31,33$.

le $\vartheta$ n 33, 34 f., 38, 39.

leףns 33, 34, 38, 39.

lusl 31 .

mae 11,39 .

mar $3,28,29,32$ f.

mari $3,28,29$ f., 30, 33 . marisl 36.

n 36 .

ne 33.

ne $\vartheta$

satres 31 .

selva $3,31,38,39$.

tecvm 38,39 .

tin $3,4,5,7 \mathrm{f} ., 10,11$.

tins $3,4,5$.

tins $\vartheta 3,4,5,8,9,12,19,28,36$.

thuse $28 \mathrm{f}$., 31 .

tlusev 28, 38, 40 .

tv $\vartheta 35$.

$\vartheta 26$.

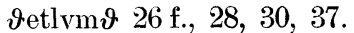

ฯne 39.

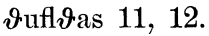

भvf $7,8,10,11,12,21$.

uni $11,38,39$.

vel 31 .

vetisl 22 f., 23,24 . 


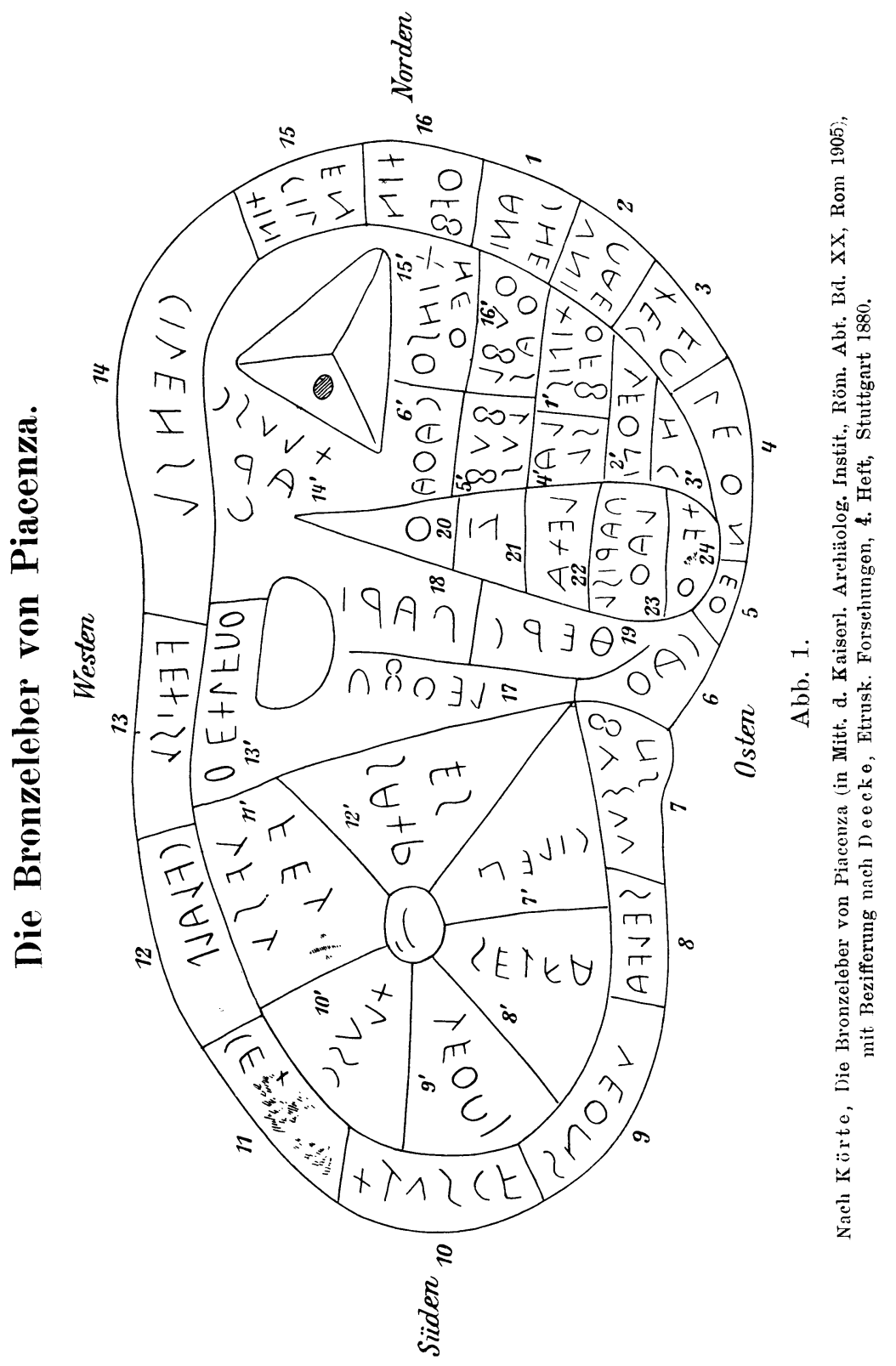




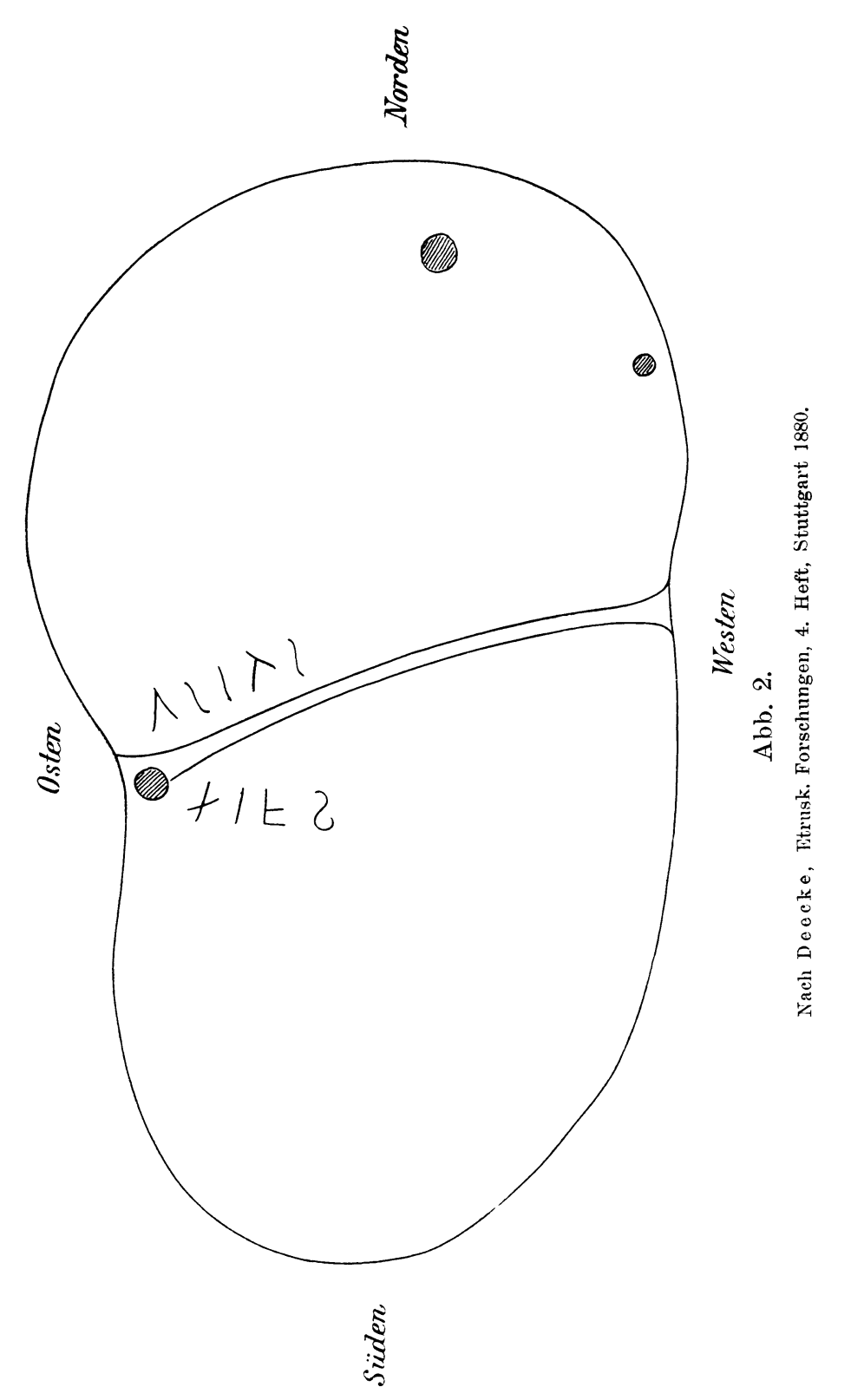

